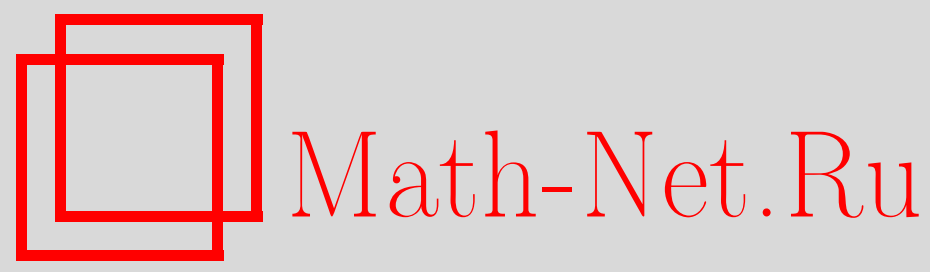

Г. Г. Амосов, А. В. Булинский, М. Е. Широков, Регулярные полугруппы эндоморфизмов факторов Неймана, Мaтем. заметки, 2001, том 70, выпуск 5, 643-659

DOI: https://doi.org/10.4213/mzm777

Использование Общероссийского математического портала Math-Net.Ru подразумевает, что вы прочитали и согласны с пользовательским соглашением http://www.mathnet.ru/rus/agreement

Параметры загрузки:

IP : 54.89 .56 .158

26 апреля 2023 г., 16:46:04

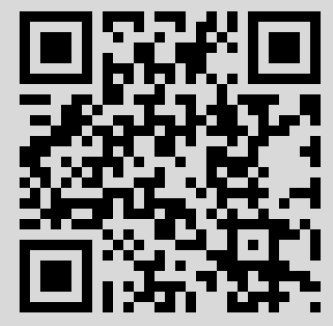




\title{
РЕГУЛЯРНЫЕ ПОЛУГРУППЫ ЭНДОМОРФИЗМОВ ФАКТОРОВ НЕЙМАНА
}

\author{
Г.Г. Амосов, А.В. Булинский, М. Е. Широков
}

Изучается класс $E_{0}$-полугрупп эндоморфизмов фактора фон Неймана $\mathscr{M}$, с каждой из которых могут быть ассоциированы $e_{0}$-полугруппа эндоморфизмов $\mathscr{B}(\mathscr{H})$, где $\mathscr{H}$ пространство стандартного представления $\mathscr{M}$, и система-произведение гильбертовых пространств.

Библиография: 16 названий.

1. Введение. Теория полугруп эндоморфизмов фактора $\mathscr{B}(\mathscr{H})$, возникшая в середине 80-х годов как обобщение теории изометрических полугрупп и симметрических операторов в гильбертовом пространстве, получила значительное развитиев последнее десятилетие благодаря работам Р. Пауэрса, У. Арвесона, Дж. Прайса, Р. Бхата, многих других исследователей (см., например, [1], [2] и цитированную там литературу). Представляет интерес развитие подобной теории для общих алгебр фон Неймана $\mathscr{R}$, в первую очередь, для факторов (алгебр с тривиальным центром: $\mathscr{R} \cap \mathscr{R}^{\prime}=\mathbb{C} I$ ). Возможность распространения элементов этой теории для определенньх полугрупा на гиперфинитных факторах рассматривалась нами в [3]. Подход был основан на естественном продолжении исходной “квазисвободной” полугрупшы эндоморфизмов с подфактора на соответствующую алгебру $\mathscr{B}(\mathscr{H})$. В данной работе мы расширяем рамки такого подхода, отправляясь от произвольного фактора фон Неймана $\mathscr{M}$ в стандартной форме в гильбертовом пространстве $\mathscr{H}$ (см. [4], [5]) и от произвольной $E_{0}$-полугрупшы $\left\{\alpha_{t}\right\}_{t \geqslant 0}$

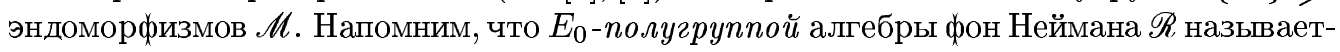
ся семейство $\left\{\alpha_{t}\right\}_{t \geqslant 0}$ ее нормальных $*$-эндоморфизмов со свойствами:

1. $\alpha_{0}=I d, \alpha_{t} \circ \alpha_{s}=\alpha_{t+s} \forall t, s \geqslant 0$;

2. функция $t \mapsto \omega\left(\alpha_{t}(A)\right)$ непрерьвна на $[0,+\infty) \forall \omega \in \mathscr{R}_{*}, \forall A \in \mathscr{R} \quad$ ( $\sigma$-слабая непрерывность);

3. $\alpha_{t}(I)=I \forall t \geqslant 0$ (унитальность, или консервативность).

При отказе от свойства 3 говорят о $e_{0}$-nолугрупnе алгебры $\mathscr{R}$ (см. [2]).

Модулярная инволющия $J$ определяет антилинейный изоморфизм $j(\cdot)=J(\cdot) J$ из $\mathscr{M}$ на $\mathscr{M}^{\prime}$, который “отражает” $E_{0}$-полугруппу $\left\{\alpha_{t}\right\}_{t \geqslant 0}$ в $E_{0}$-полугруппу

$$
\left\{\beta_{t}=j \circ \alpha_{t} \circ j^{-1}\right\}_{t \geqslant 0}
$$

на коммутанте $\mathscr{M}^{\prime}$. В [3] было введено понятие регулярного расширения полугруппы $\left\{\alpha_{t}\right\}_{t \geqslant 0}$ как такой $E_{0}$-полугруппы $\left\{\theta_{t}\right\}_{t \geqslant 0}$ эндоморфизмов $\mathscr{B}(\mathscr{H})$, что

$$
\theta_{t}(A)=\alpha_{t}(A), \quad \theta_{t}(B)=\beta_{t}(B) \quad \forall A \in M, \quad \forall B \in M^{\prime}, \quad \forall t \geqslant 0 .
$$

(C) Г.Г. Амосов, А.В. Булинский, М.Е. Широков 2001 
В силу слабой плотности в $\mathscr{B}(\mathscr{H})$ *-алгебры, порожденной факторами $\mathscr{M}$ и $\mathscr{M}^{\prime}$, регулярное расширение однозначно определяется полугруппй $\left\{\alpha_{t}\right\}_{t \geqslant 0}$, а для существования такого расширения $\left\{\theta_{t}\right\}_{t \geqslant 0}$ необходимо и достаточно, чтобы при некотором $t>0$ (a значит и при всех $t \geqslant 0)$ алгебра фон Неймана, порожденная факторами $\alpha_{t}(\mathscr{M})$ и $\beta_{t}\left(\mathscr{M}^{\prime}\right)$, являлась фактором. Вопрос о том, будет ли фактором пересечение или "объединение" двух факторов, восходит еще к фон Нейману, которьй показал, что это заведомо имеет место для факторов типа I. Таким образом, регулярное расширение всегда существует, если $\mathscr{M}$ - фактор типа I. Однако регулярное расширение может существовать и для полугрупп эндоморфизмов на факторах типа II и III. В работе [3] явно построено регулярное расширение для квазисвободных $E_{0}$-полугрупп на гиперфинитных факторах типа $\mathrm{II}_{1}$ и $\mathrm{III}_{\lambda}, 0<\lambda<1$, порожденных представлениями $C^{*}$-алгебры канонических антикоммутационных соотношений (KAC).

Ниже рассматривается замкнутьй относительно коциклических возмущений класс полугрупп на факторе $\mathscr{M}$ произвольного типа, названных регулярнылми, для которых существует "обобщенное решение" задачи о регулярном расширении. Именно, в разделе 3 показано, что с каждой регулярной полугруппой $\left\{\alpha_{t}\right\}_{t \geqslant 0}$ может быть связана определенная $e_{0}$-полугруппа эндоморфизмов $\mathscr{B}(\mathscr{H})$, которая совпадает с регулярным расширением, если такое расширение существует. Возникающая $e_{0}$-полугрупп $\left\{\bar{\theta}_{t}\right\}_{t \geqslant 0}$ названа регулярным квазирасиирением на $\mathscr{B}(\mathscr{H})$ исходной $E_{0}$-полугрупшы $\left\{\alpha_{t}\right\}_{t \geqslant 0}$. Предварительно в разделе 2 изучается случай отдельного эндоморфизма и для него вводится понятие регулярности и регулярного квазирасширения на $\mathscr{B}(\mathscr{H})$.

Исследование существования регулярного расширения на $\mathscr{B}(\mathscr{H})$ тесно связано с изучением семейства $\left\{\mathscr{E}_{\alpha}(t)\right\}_{t \geqslant 0}$ гильбертовых пространств, где при каждом $t \geqslant 0$

$$
\mathscr{E}_{\alpha}(t)=\left\{X \in \mathscr{B}(\mathscr{H}): X A=\alpha_{t}(A) X \quad \forall A \in \mathscr{M}, \quad X B=\beta_{t}(B) X \quad \forall B \in \mathscr{M}^{\prime}\right\},
$$

а скалярное произведение $\langle\cdot, \cdot\rangle_{\mathscr{E}_{\alpha}(t)}$ определяется равенством $X^{*} Y=\langle X, Y\rangle_{\mathscr{E}_{\alpha}(t)} I$ $\forall X, Y \in \mathscr{E}_{\alpha}(t)$.

Необходимым условием существования регулярного расширения $\left\{\theta_{t}\right\}_{t \geqslant 0}$ является наличие у семейства $\left\{\mathscr{E}_{\alpha}(t)\right\}_{t \geqslant 0}$ свойства системы-произведения (СП) гильбертовых пространств с операторньм умножением в качестве тензорного произведения “слоев". Тогда данная СП является ассоциированной по Арвесону с $E_{0}$-полугруппой эндоморфизмов $\left\{\theta_{t}\right\}_{t \geqslant 0}[6]$. Подробный анализ СП гильбертовых пространств в связи с теорией индекса $E_{0}$-полугрупп $\mathscr{B}(\mathscr{H})$ проведен в фундаментальной работе [6]. Наиболее полные результаты относятся к случаю полугруп, вполне пространственных по терминологии Пауэрса. Некоторые обобщения можно найти в работе Бхата [7]. Мы вынуждены опустить соответствующие точные определения, адресуя читателя к литературе. Отметим только, что СП $\{\mathscr{E}(t)\}_{t \geqslant 0}$ (введенная как непрерывньй аналог фоковского пространства) состоит из гильбертовых пространств - “слоев" $\mathscr{E}(t)$, при тензорном умножении которых вьполняется соотношение $\mathscr{E}(t) \otimes \mathscr{E}(s) \cong \mathscr{E}(t+s) \forall t, s \geqslant 0$.

В нашей работе установлено необходимое и достаточное условие, при котором семейство $\left\{\mathscr{E}_{\alpha}(t)\right\}_{t \geqslant 0}$ является СП гильбертовых пространств. В разделе 3 также показано, что при каждом $t \geqslant 0$ в пространстве $\mathscr{E}_{\alpha}(t)$ существует наибольшее нетривиальное подпространство $\overline{\mathscr{E}}_{\alpha}(t)$ такое, что семейство $\left\{\overline{\mathscr{E}}_{\alpha}(t)\right\}_{t \geqslant 0}$ является СП. В разделе 4 показана устойчивость рассматриваемых объектов по отношению к коциклическим возмущениям полугрупшы $\left\{\alpha_{t}\right\}_{t \geqslant 0}$. Ситуация проиллюстрирована в разделе 5 на более общем, чем в [3], примере квазисвободных эндоморфизмов алгебры КАС. 
Используются следующие обозначения, в основном соответствуюшие [5]: $I$ - единичньй оператор в $\mathscr{H}, \operatorname{End}(\mathscr{R})$ - множество всех нормальных *-эндоморфизмов алгебры фон Неймана $\mathscr{R}$, т.е. $\sigma\left(\mathscr{R}, \mathscr{R}_{*}\right)$-непрерьвных $*$-морфизмов из $\mathscr{R}$ в $\mathscr{R}, \mathscr{R}_{*}-$ преддвойственное пространство нормальных линейных функционалов на $\mathscr{R}, \mathscr{R}_{+}-$положительный конус в $\mathscr{R}$. Если $\mathscr{R}_{1}$ и $\mathscr{R}_{2}$ алгебры фон Неймана в $\mathscr{H}$, то $\mathscr{R}_{1} \wedge \mathscr{R}_{2} \equiv \mathscr{R}_{1} \cap \mathscr{R}_{2}$. Под $\mathscr{R}_{1} \vee \mathscr{R}_{2}$ будем понимать не алгебру фон Неймана, а $*$-алгебру, ими порожденную, и ее слабое замыкание будем обозначать $\left[\mathscr{R}_{1} \vee \mathscr{R}_{2}\right]^{-}$. Символ $\mathscr{R}_{1} \bar{\otimes}_{2}$ обозначает алгебру фон Неймана, порожденную (алгебраическим) тензорным произведением $\mathscr{R}_{1} \otimes \mathscr{R}_{2}$. Символ $\left.(\cdot)\right|_{\mathscr{K}}$ будет, как обычно, обозначать сужение оператора или алгебры на подпространство $\mathscr{K} \subseteq \mathscr{H}$. Для ортогональных проекторов $P$ и $Q$ в $\mathscr{H}$ верхнюю и нижнюю грань обозначаем $P \vee Q$ и $P \wedge Q$ соответственно. Аналогичные символы используются и для соответствующих подпространств в $\mathscr{H}:(P \vee Q) \mathscr{H}=P \mathscr{H} \vee Q \mathscr{H}$, $(P \wedge Q) \mathscr{H}=P \mathscr{H} \wedge Q \mathscr{H}$.

2. Случай одного эндоморфизма. Пусть $\alpha$ - эндоморфизм фактора $\mathscr{M}$, стандартно реализованного в гильбертовом пространстве $\mathscr{H}, \Omega$ - некоторьй бициклический для $\mathscr{M}$ вектор и $J=J_{\Omega}$ - ассоциированная с $\Omega$ модулярная инволюция. Полагая $j(\cdot)=J(\cdot) J$, обозначим $\beta=j \circ \alpha \circ j^{-1} \in \operatorname{End}\left(\mathscr{M}^{\prime}\right)$ и рассмотрим множество

$$
\mathscr{E}_{\alpha}^{\Omega}=\left\{X \in \mathscr{B}(\mathscr{H}): X A=\alpha(A) X \quad \forall A \in \mathscr{M}, \quad X B=\beta(B) X \quad \forall B \in \mathscr{M}^{\prime}\right\} .
$$

В силу $\left(\mathscr{M} \vee \mathscr{M}^{\prime}\right)^{\prime}=\mathbb{C} I$ множество $\mathscr{E}_{\alpha}^{\Omega}$ есть гильбертово пространство со скалярным произведением $\langle\cdot, \cdot\rangle_{\mathscr{E}_{\alpha}^{\Omega}}$, определяемым равенством $X^{*} Y=\langle X, Y\rangle_{\mathscr{E}_{\alpha}} I$. Доказательство этого утверждения аналогично данному в [6] для множества операторов, сплетающих эндоморфизм $\mathscr{B}(\mathscr{H})$.

Если выбрать вместо $\Omega$ другой бициклический вектор $\Omega^{\prime}$, то в случае $J_{\Omega^{\prime}} \neq J_{\Omega}$ изменится эндоморфизм $\beta$, а значит, вообще говоря, $\mathscr{E}_{\alpha} \Omega^{\prime} \neq \mathscr{E}_{\alpha} \Omega$. "Независимость" от $\Omega$ показывает следующая

Лемма 1. Если $\Omega^{\prime}$ отличный от $\Omega$ бичиклический вектор для $\mathscr{M}$, то гильбертовы пространства $\mathscr{E}_{\alpha}$ и $\mathscr{E}_{\alpha} \Omega^{\prime}$ изоморфны. Роль унитарного преобразования из $\mathscr{E}_{\alpha} \Omega$ на $\mathscr{E}_{\alpha}^{\Omega^{\prime}}$ играет отображсние $A d_{W^{*}}=W^{*}(\cdot) W$, где $W$ - унитарный оператор, принадлежсаий $\mathscr{M}^{\prime}$.

ДокАЗАТЕЛЬСТво. Если $\Omega^{\prime}$ лежит в ассоциированном с $\Omega$ положительном конусе $\mathscr{P}_{\Omega}$, то в силу предложения 2.5 .30 из [4] $J_{\Omega^{\prime}}=J_{\Omega}$, а значит, $\mathscr{E}_{\alpha} \Omega^{\prime}=\mathscr{E}_{\alpha} \Omega$ и утверждение леммы получается при $W=I$. Если $\Omega^{\prime}$ не лежит в $\mathscr{P}_{\Omega}$, то по основному свойству конуса $\mathscr{P}_{\Omega}$ в нем найдется бициклический вектор $\Omega^{\prime \prime}$ такой, что $\left(\Omega^{\prime}, A \Omega^{\prime}\right)=\left(\Omega^{\prime \prime}, A \Omega^{\prime \prime}\right)$ $\forall A \in \mathscr{M}$. Следовательно, соответствие $A \Omega^{\prime} \mapsto A \Omega^{\prime \prime}$ определяет унитарньй оператор $W \in \mathscr{M}^{\prime}$. Если, как обычно, $S_{\Omega}$ - антилинейный оператор, задаваемьй условием $S_{\Omega} A \Omega=A^{*} \Omega \forall A \in \mathscr{M}$, то для операторов $S_{\Omega^{\prime}}$ и $S_{\Omega^{\prime \prime}}$ имеем

$$
S_{\Omega^{\prime \prime}} W A \Omega^{\prime}=S_{\Omega^{\prime \prime}} A \Omega^{\prime \prime}=A^{*} \Omega^{\prime \prime}=W A^{*} \Omega^{\prime}=W S_{\Omega^{\prime}} A \Omega^{\prime} .
$$

Поэтому $S_{\Omega^{\prime}}=W^{*} S_{\Omega^{\prime \prime}} W$. Единственность полярного разложения дает $J_{\Omega^{\prime}}=W^{*} J_{\Omega^{\prime \prime}} W$. Но $J_{\Omega^{\prime \prime}}=J_{\Omega}=J$ в силу $\Omega^{\prime \prime} \in \mathscr{P}_{\Omega}$ и предложения 2.5.30 из [4]. Используя то, что $J^{\prime}=J_{\Omega^{\prime}}=W^{*} J_{\Omega} W=W^{*} J W$ и $W \in \mathscr{M}^{\prime}$, получаем $\forall B \in \mathscr{M}^{\prime}$

$$
\begin{aligned}
\beta^{\prime}(B) & :=J^{\prime} \alpha\left(J^{\prime} B J^{\prime}\right) J^{\prime}=W^{*} J W \alpha\left(W^{*} J W B W^{*} J W\right) W^{*} J W \\
& =W^{*} J \alpha\left(J W B W^{*} J\right) J W=W^{*} \beta\left(W B W^{*}\right) W .
\end{aligned}
$$


Непосредственная проверка с учетом (3) показьвает, что $W^{*}(\cdot) W$ отображает множество $\mathscr{E}_{\alpha} \Omega$ на множество $\mathscr{E}_{\alpha}^{\Omega^{\prime}}$ (которое определяется $(2)$ с заменой $\beta$ на $\beta^{\prime}$ ).

Далее, для любых $X$ и $Y$ из $\mathscr{E}_{\alpha} \Omega$ имеем

$$
\begin{aligned}
\left\langle W^{*} X W, W^{*} Y W\right\rangle_{\mathscr{E}_{\alpha}^{\Omega^{\prime}}} I & =W^{*} X^{*} W W^{*} Y W=W^{*} X^{*} Y W \\
& =W^{*}\langle X, Y\rangle_{\mathscr{E}_{\alpha} \Omega} I W=\langle X, Y\rangle_{\mathscr{E}_{\alpha}} I .
\end{aligned}
$$

Таким образом, $W^{*}(\cdot) W$ - унитарное преобразование из $\mathscr{E}_{\alpha}^{\Omega}$ на $\mathscr{E}_{\alpha} \Omega^{\prime}$.

В силу леммы 1 можно опустить индекс $\Omega$ в обозначении гильбертова пространства: $\mathscr{E}_{\alpha} \Omega=\mathscr{E}_{\alpha}$.

Обозначим через $\mathscr{R}$ алгебру фон Неймана, порожденную факторами $\alpha(\mathscr{M})$ и $\beta\left(\mathscr{M}^{\prime}\right)$, т.е. $\mathscr{R}=\left[\alpha(\mathscr{M}) \vee \beta\left(\mathscr{M}^{\prime}\right)\right]^{-}$. Тогда $\mathscr{R}^{\prime}=\alpha(\mathscr{M})^{\prime} \wedge \beta\left(\mathscr{M}^{\prime}\right)^{\prime}$.

ЛЕмма 2. Пусть изометрия $T$ принадлежит $\mathscr{E}_{\alpha}$. Тогда проектор ТT ${ }^{*}$ является минимальным в алгебре $\mathscr{R}^{\prime}$.

ДокАЗАТЕЛьСтво. Заметим, что $E=T T^{*} \in \mathscr{R}^{\prime}$, поскольку $T T^{*} \alpha(A)=T A T^{*}=$ $\alpha(A) T T^{*} \forall A \in \mathscr{M}$ и $T T^{*} \beta(B)=T B T^{*}=\beta(B) T T^{*} \forall B \in \mathscr{M}^{\prime}$ в силу $T \in \mathscr{E}_{\alpha}$. Алгебpa $\left.\left(E \mathscr{R}^{\prime} E\right)\right|_{E \mathscr{H}}$ является по предложению 5.5.6 из [5] коммутантом в $\mathscr{B}(E \mathscr{H})$ алгебры $\left.(E \mathscr{R})\right|_{E \mathscr{H}}=\left.\left(T \mathscr{B}(\mathscr{H}) T^{*}\right)\right|_{E \mathscr{H}}=\mathscr{B}(E \mathscr{H})$. Поэтому $E \mathscr{R}^{\prime} E=\mathbb{C} E$ и $E$ минимален в $\mathscr{R}^{\prime}$.

Лемма 1 позволяет ввести следующее

ОПРЕДЕЛЕНИЕ 1. Эндоморфизм $\alpha \in \operatorname{End}(\mathscr{M})$ назьвается регулярнылм, если

$$
\operatorname{dim} \mathscr{E}_{\alpha}>0
$$

Назовем регулярным подрасширением эндоморфизма $\alpha$ фактора $\mathscr{M}$ такой эндоморфизм $\varphi$ фактора $\mathscr{B}(\mathscr{H})$, что $0 \neq \varphi(I) \in \mathscr{R}^{\prime}$ и

$$
\varphi(A)=\varphi(I) \alpha(A) \quad \forall A \in \mathscr{M}, \quad \varphi(B)=\varphi(I) \beta(B) \quad \forall B \in \mathscr{M}^{\prime} .
$$

Множество $\Phi(\alpha) \subset \operatorname{End}(\mathscr{B}(\mathscr{H}))$ всех регулярных подрасширений эндоморфизма $\alpha$ непусто тогда и только тогда, когда $\alpha$ - регулярный эндоморфизм. Действительно, любая изометрия $T$ из $\mathscr{E}_{\alpha}$ дает регулярное подрасширение $T(\cdot) T^{*}$, поскольку $T T^{*} \in \mathscr{R}^{\prime}$ и

$$
T A T^{*}=T T^{*} \alpha(A) \quad \forall A \in \mathscr{M}, \quad T B T^{*}=T T^{*} \beta(B) \quad \forall B \in \mathscr{M}^{\prime} .
$$

Наоборот, пусть $\varphi$ - регулярное подрасширение эндоморфизма $\alpha$. Согласно [6] для всякого $\varphi \in \operatorname{End}(\mathscr{B}(\mathscr{H}))$ найдется хотя бы одна (сплетающая) изометрия $T$ такая, что $T X=\varphi(X) T$. Легко видеть, что оператор $T \in \mathscr{E}_{\alpha}$.

ЛЕмма 3. Сужсение любого $\varphi \in \Phi(\alpha)$ на *-алгебру $\mathscr{M} \vee \mathscr{M}^{\prime}$ совпадает с отображением

$$
\sum_{k} A_{k} B_{k} \mapsto \varphi(I)\left[\sum_{k} \alpha\left(A_{k}\right) \beta\left(B_{k}\right)\right],
$$

где $\left\{A_{k}\right\}$ и $\left\{B_{k}\right\}$ - конечные наборы әлементов из $\mathscr{M} и \mathscr{M}^{\prime}$ соответственно. 
ДокАЗАТЕЛЬСтво. Проверяется непосредственно с учетом определения эндоморфизма $\varphi$.

Во множестве $\operatorname{End}(\mathscr{B}(\mathscr{H}))$ существует естественное отношение частичного порядка: $\gamma_{1} \succ \gamma_{2} \Leftrightarrow \gamma_{1}(X) \geqslant \gamma_{2}(X) \forall X \in(\mathscr{B}(\mathscr{H}))_{+}$.

Лемма 4. Если $\varphi_{1}, \varphi_{2} \in \Phi(\alpha)$, mo $\varphi_{1} \succ \varphi_{2} \Leftrightarrow \varphi_{1}(I) \geqslant \varphi_{2}(I)$.

ДокАЗАТЕЛЬСТво. Утверждение леммы следует из сильной плотности *-алгебры $\mathscr{M} \vee \mathscr{M}^{\prime}$ в $\mathscr{B}(\mathscr{H})$ и леммы 3.

ТЕОрема 1. Множество $\Phi(\alpha)$ всех регулярных подрасиирений регулярного эндоморфизма $\alpha$ содержит наибольший әлемент $\theta$. Эндоморфизм $\theta$ имеет вид

$$
\theta(\cdot)=\sum_{i=1}^{n} T_{i}(\cdot) T_{i}^{*}
$$

әде $\left\{T_{i}\right\}_{i=1}^{n}-$ произвольный ортонормированный базис (ОНБ) в $\mathscr{E}_{\alpha}, n=\operatorname{dim} \mathscr{E}_{\alpha} \leqslant \infty$.

Проектор $C=\theta(I)$ является минимальным в $\mathscr{R} \wedge \mathscr{R}^{\prime}$. Мнохество $\mathscr{E}_{\alpha}$ есть гильбертово пространство операторов, сплетающих әндоморфизм $\theta$.

ДокАЗАТЕЛЬСТво. Пусть $\left\{T_{i}\right\}_{i=1}^{n}-$ произвольньй ОНБ в $\mathscr{E}_{\alpha}$, т.е. семейство изометрий со взаимно ортогональными образами, дающими в объединении подпространство $\bigvee_{X \in \mathscr{E}_{\alpha}} \operatorname{Ran} X$. Эндоморфизм $\theta(\cdot)=\sum_{i=1}^{n} T_{i}(\cdot) T_{i}^{*}$ является регулярньм подрасширением $\alpha$, поскольку

$$
\begin{aligned}
& \theta(A)=\sum_{i=1}^{n} T_{i} A T_{i}^{*}=\alpha(A) \sum_{i=1}^{n} T_{i} T_{i}^{*}=\alpha(A) \theta(I) \quad \forall A \in \mathscr{M}, \\
& \theta(B)=\sum_{i=1}^{n} T_{i} B T_{i}^{*}=\beta(B) \sum_{i=1}^{n} T_{i} T_{i}^{*}=\beta(B) \theta(I) \quad \forall B \in \mathscr{M}^{\prime} .
\end{aligned}
$$

Пусть $\varphi-$ произвольное подрасширение $\alpha$. Для $\varphi \in \operatorname{End}(\mathscr{B}(\mathscr{H}))$ имеем

$$
\varphi(\cdot)=\sum_{i=1}^{m} U_{i}(\cdot) U_{i}^{*},
$$

где $\left\{U_{i}\right\}_{i=1}^{m}-$ некоторое семейство изометрий со взаимно ортогональньми образами, сплетаюших $\varphi$ (см. [6]). Так как $\varphi$ является подрасширением $\alpha$, то при каждом $k \leqslant m$

$$
\begin{aligned}
& U_{k} A=\varphi(A) U_{k}=\alpha(A) \varphi(I) U_{k}=\alpha(A)\left(\sum_{i=1}^{m} U_{i} U_{i}^{*}\right) U_{k}=\alpha(A) U_{k} \quad \forall A \in \mathscr{M}, \\
& U_{k} B=\varphi(B) U_{k}=\beta(B) \varphi(I) U_{k}=\beta(B)\left(\sum_{i=1}^{m} U_{i} U_{i}^{*}\right) U_{k}=\beta(B) U_{k} \quad \forall B \in \mathscr{M}^{\prime} .
\end{aligned}
$$

Следовательно, $U_{k} \in \mathscr{E}_{\alpha}$ и $\varphi(I)=\sum_{i=1}^{m} U_{i} U_{i}^{*} \leqslant C=\theta(I)$. Лемма 4 дает $\varphi \prec \theta$. Таким образом, максимальность $\theta$ доказана.

Покажем, что $C=\theta(I) \in \mathscr{R} \wedge \mathscr{R}^{\prime}$. Ясно, что $C=\theta(I) \in \mathscr{R}^{\prime}$. Поскольку $\left.\left(C \mathscr{R}^{\prime} C\right)\right|_{C \mathscr{H}}$ есть коммутант в $\mathscr{B}(C \mathscr{H})$ фактора $\left.(C \mathscr{R})\right|_{C \mathscr{H}}=\left.(\theta(\mathscr{B}(\mathscr{H})))\right|_{C \mathscr{H}}$ типа I, то $C \mathscr{R}^{\prime} C-$ 
фактор типа I. Пусть $T$ - произвольная изометрия из $\mathscr{E}_{\alpha}$. Тогда по лемме 2 проектор $E=T T^{*}$ является минимальным в алгебре $\mathscr{R}^{\prime}$ и $E \leqslant C$. Поскольку $C \mathscr{R}^{\prime} C$ - фактор, то $C \leqslant C_{E}$, где $C_{E}$ - центральньй носитель проектора $E$ в алгебре $\mathscr{R}^{\prime}$. Предположим, что $C<C_{E}$. Тогда в факторе $C_{E} \mathscr{R}^{\prime}$ типа I найдется частичная изометрия $V$ такая, что $V^{*} V=E$ и $V V^{*} \leqslant C_{E}-C$. Для изометрии $V T$ имеем $V T A=V \alpha(A) T=\alpha(A) V T$ $\forall A \in \mathscr{M}$ и $V T B=V \beta(B) T=\beta(B) V T \forall B \in \mathscr{M}^{\prime}$, т.е. $V T \in \mathscr{E}_{\alpha}$. Следовательно, $V T(V T)^{*}=V V^{*} \leqslant C$. Полученное противоречие показывает, что $C=C_{E} \in \mathscr{R} \wedge \mathscr{R}^{\prime}$. Минимальность $C=C_{E}$ в $\mathscr{R} \wedge \mathscr{R}^{\prime}$ следует из минимальности $E$ в $\mathscr{R}^{\prime}$ и предложения 6.4.3 из [5].

ЗАмЕчАнИЕ 1. Из (алгебраической) изоморфности алгебр $\mathscr{M} \vee \mathscr{M}^{\prime}$ и $\mathscr{M} \otimes \mathscr{M}^{\prime}$ (теорема 5.5.4 в [5]) следует, что сопоставление конечных сумм

$$
\sum_{k} A_{k} B_{k} \mapsto \sum_{k} \alpha\left(A_{k}\right) \beta\left(B_{k}\right)
$$

корректно определяет инъективньй ж-гомоморфизм алгебры $\mathscr{M} \vee \mathscr{M}^{\prime}$ в себя, расширяющий $\alpha$ и $\beta$. Для его расширения до эндоморфизма $\mathscr{B}(\mathscr{H})$ необходима и достаточна ограниченность и $\sigma$-слабая непрерьвность этого гомоморфизма (лемма 10.11 из [5]). Теорема 1 и лемма 3 показьвают, что в $\mathscr{H}$ сушествует наибольшее подпространство $C \mathscr{H}$, приводящее факторы $\alpha(\mathscr{M})$ и $\beta\left(\mathscr{M}^{\prime}\right)$, такое, что отображение

$$
\left.\sum_{k} A_{k} B_{k} \mapsto\left[\sum_{k} \alpha\left(A_{k}\right) \beta\left(B_{k}\right)\right]\right|_{C \mathscr{H}}
$$

ограничено и $\sigma$-слабо непрерьвно.

В случае $C=\theta(I)=I$, т.е. когда $\bigvee_{X \in \mathscr{E}_{\alpha}} \operatorname{Ran} X=\mathscr{H}$, эндоморфизм $\theta$ является регулярным расиирением эндоморфизма $\alpha$ (см. [3]), т.е.

$$
\theta(A)=\alpha(A), \quad \theta(B)=\beta(B) \quad \forall A \in \mathscr{M}, \quad \forall B \in \mathscr{M}
$$

Наоборот, если регулярное расширение существует, то оно, очевидно, совпадает с $\theta$. Поэтому в общем случае $C=\theta(I) \leqslant I$ эндоморфизм $\theta$ будем называть регулярным квазирасширением эндоморфизма $\alpha$.

Подчеркнем, что если $\mathscr{M}$ - фактор типа I, то любой эндоморфизм $\alpha$ является регулярным и его регулярное квазирасширение унитально: $C=\theta(I)=I$. Действительно, согласно [6] в этом случае $\alpha(\cdot)=\sum_{i=1}^{n} V_{i}(\cdot) V_{i}^{*}$, где $\left\{V_{i}\right\}_{i=1}^{n}-$ семейство изометрий из $\mathscr{M}$. Нетрудно проверить, что семейство $\left\{V_{i, j}=V_{i} J V_{j} J\right\}_{i, j=1}^{n}$ есть ОНБ в $\mathscr{E}_{\alpha}$ и $\theta(I)=\sum_{i, j=1}^{n} V_{i, j} V_{i, j}^{*}=I$. Если $\mathscr{M}$ - фактор типа II или III, то регулярные эндоморфизмы существуют и их регулярные квазирасширения также могут быть унитальными, как показано в [3] и в примерах раздела 5.

Отметим, что рассуждения раздела 2 можно провести в терминах дискретной полугруппы $\left\{\alpha^{n}\right\}_{n \in \mathbb{Z}_{+}}$, где $\alpha$ - регулярньй эндоморфизм $\mathscr{M}$, и соответствующего регулярного квазирасширения - полугруппы $\left\{\theta^{n}\right\}_{n \in \mathbb{Z}_{+}}$.

3. Расширение $E_{0}$-полугруппы эндоморфизмов. Пусть $E_{0}$-полугрупа

$$
\left\{\alpha_{t}\right\}_{t \geqslant 0}
$$


состоит из регулярных эндоморфизмов фактора $\mathscr{M}$, a $\left\{\beta_{t}=j \circ \alpha_{t} \circ j^{-1}\right\}_{t \geqslant 0}-$ соответствующая полугруппа на $\mathscr{M}^{\prime}$. Результаты предыдущего раздела позволяют при каждом $t \geqslant 0$ ввести гильбертово пространство $\mathscr{E}_{\alpha}(t):=\mathscr{E}_{\alpha_{t}}$, определяемое $(1)$, и регулярное квазирасширение $\theta_{t}$. Естественньй вопрос о наличии у семейства $\left\{\theta_{t}\right\}_{t \geqslant 0}$ свойства $E_{0}$-полугруппы $\mathscr{B}(\mathscr{H})$ равносилен вопросу о том, образует ли семейство $\left\{\mathscr{E}_{\alpha}(t)\right\}_{t \geqslant 0}$ СП гильбертовых пространств по Арвесону (см. [6]) с операторньп умножением в $\mathscr{B}(\mathscr{H})$ в качестве тензорного произведения слоев $\mathscr{E}_{\alpha}(t)$. Легко проверить, что $X_{t} X_{s} \in \mathscr{E}_{\alpha}(t+s)$ $\forall X_{t} \in \mathscr{E}_{\alpha}(t), \forall X_{s} \in \mathscr{E}_{\alpha}(s)$, т.е. что $\mathscr{E}_{\alpha}(t) \mathscr{E}_{\alpha}(s) \subseteq \mathscr{E}_{\alpha}(t+s)$, однако априори не очевидно, будет ли линейная оболочка множества $\mathscr{E}_{\alpha}(t) \mathscr{E}_{\alpha}(s)$ плотна в $\mathscr{E}_{\alpha}(t+s)$. Как показано ниже, данньй вопрос тесно связан с поведением семейства проекторов $\left\{C_{t}=\theta_{t}(I)\right\}_{t \geqslant 0}$.

Лемма 5. Семейство проекторов $\left\{C_{t}=\theta_{t}(I)\right\}_{t \geqslant 0}$ коммутативно и

$$
\theta_{t}\left(C_{s}\right)=C_{t} C_{t+s}
$$

ДоКАЗАТЕЛЬСТво. Коммутативность семейства $\left\{C_{t}\right\}_{t \geqslant 0}$ легко выводится из доказанной в теореме 1 принадлежности при каждом $t>0$ проектора $C_{t}$ алгебре $\mathscr{R}_{t} \wedge \mathscr{R}_{t}^{\prime}$ и очевидных включений $\mathscr{R}_{t} \supseteq \mathscr{R}_{t+s}, \mathscr{R}_{t}^{\prime} \subseteq \mathscr{R}_{t+s}^{\prime} \forall t, s>0$. Поскольку $\theta_{t}\left(\alpha_{s}(A) \beta_{s}(B)\right)=$ $C_{t} \alpha_{t+s}(A) \beta_{t+s}(B) \forall A \in \mathscr{M}, \forall B \in \mathscr{M}^{\prime}$ в силу леммы 3 , то

$$
\theta_{t}\left(\mathscr{R}_{s}\right)=C_{t} \mathscr{R}_{t+s}
$$

Из $(4)$ с учетом $\theta_{t}(\mathscr{B}(\mathscr{H}))=C_{t} \theta_{t}(\mathscr{B}(\mathscr{H}))$ получаем

$$
\theta_{t}\left(\mathscr{R}_{s}^{\prime}\right) \subseteq C_{t} \mathscr{R}_{t+s}^{\prime} C_{t}
$$

Из (4) и (5) следует, что $\theta_{t}\left(\mathscr{R}_{s} \wedge \mathscr{R}_{s}^{\prime}\right) \subseteq C_{t}\left(\mathscr{R}_{t+s}^{\prime} \wedge \mathscr{R}_{t+s}^{\prime}\right)$. Поскольку $C_{s} \in \mathscr{R}_{s} \wedge \mathscr{R}_{s}^{\prime}$ по теореме 1 , то $\theta_{t}\left(C_{s}\right)$ - проектор в $C_{t}\left(\mathscr{R}_{t+s}^{\prime} \wedge \mathscr{R}_{t+s}^{\prime}\right)$.

Далее, $\theta_{t}(\cdot)=\sum_{i=1}^{n_{t}} T_{t}^{i}(\cdot)\left(T_{t}^{i}\right)^{*}$, где $\left\{T_{t}^{i}\right\}_{i=1}^{n_{t}}-$ произвольный ОНБ в $\mathscr{E}_{\alpha}(t)$, а

$$
C_{s}=\sum_{j=1}^{n_{s}} T_{s}^{j}\left(T_{s}^{j}\right)^{*}
$$

где $\left\{T_{s}^{j}\right\}_{j=1}^{n_{s}}-$ произвольньй ОНБ в $\mathscr{E}_{\alpha}(s)$. Поэтому с учетом $\mathscr{E}_{\alpha}(t) \mathscr{E}_{\alpha}(s) \subseteq \mathscr{E}_{\alpha}(t+s)$ имеем

$$
\theta\left(C_{s}\right)=\sum_{i=1}^{n_{t}} \sum_{j=1}^{n_{s}} T_{t}^{i} T_{s}^{j}\left(T_{s}^{j}\right)^{*}\left(T_{t}^{i}\right)^{*} \leqslant C_{t+s}
$$

Проектор $C_{t+s}$ минимален в алгебре $\mathscr{R}_{t+s} \wedge \mathscr{R}_{t+s}^{\prime}$ по теореме 1 . Следовательно, проектор $C_{t} C_{t+s}$ минимален в алгебре $C_{t}\left(\mathscr{R}_{t+s} \wedge \mathscr{R}_{t+s}^{\prime}\right)$ (предложение 5.5.5 из [5]). Эта минимальность вместе с (6) дает $\theta_{t}\left(C_{s}\right)=C_{t} C_{t+s}$.

Следующая лемма показывает наличие ослабленной версии полугруппового свойства у семейства эндоморфизмов $\left\{\theta_{t}\right\}_{t \geqslant 0}$.

ЛЕмма 6. Эндоморфизмы семейства $\left\{\theta_{t}\right\}_{t \geqslant 0}$ связаны соотношением

$$
\theta_{t}\left(\theta_{s}(\cdot)\right)=\theta_{t}(I) \theta_{t+s}(\cdot) .
$$


ДокаЗАТЕЛЬСтво. Для любых $A \in \mathscr{M}$ и $B \in \mathscr{M}^{\prime}$ в силу лемм 3 и 5 имеем

$$
\begin{aligned}
\theta_{t}\left(\theta_{s}(A B)\right) & =\theta_{t}\left(C_{s} \alpha_{s}(A) \beta_{s}(B)\right)=\theta_{t}\left(C_{s}\right) \theta_{t}\left(\alpha_{s}(A) \beta_{s}(B)\right)=C_{t} C_{t+s} \alpha_{t+s}(A) \beta_{t+s}(B) \\
& =\theta_{t}(I) \theta_{t+s}(A B) .
\end{aligned}
$$

Утверждение леммы следует из слабой плотности в $\mathscr{B}(\mathscr{H})$ алгебры $\mathscr{M} \vee \mathscr{M}^{\prime}$ и нормальности эндоморфизмов семейства $\left\{\theta_{t}\right\}_{t \geqslant 0}$.

ОПРЕДЕЛЕНИЕ 2. $E_{0}$-полугруппа эндоморфизмов $\left\{\alpha_{t}\right\}_{t \geqslant 0}$ фактора $\mathscr{M}$, стандартно реализованного в гильбертовом пространстве $\mathscr{H}$, назьвается регулярной, если

1. существует сильно непрерьвное семейство изометрий $\left\{T_{t}\right\}_{t \geqslant 0}$ такое, что $T_{t} \in \mathscr{E}_{\alpha}(t)$ $\forall t \geqslant 0$

2. $\exists t_{0}>0: \bigwedge_{s \leqslant t_{0}} C_{s}>0$.

ЗАмЕчАниЕ 2. Второе условие в определении 2 носит технический характер. Это условие заведомо вьполнено в “унитальном" случае $C_{t}=I \forall t \geqslant 0$, а также когда семейство $\left\{T_{t}\right\}_{t \geqslant 0}$ является коммутативньм, поскольку, как показывает следующая лемма, в этом случае $\operatorname{Ran} T_{t} \subseteq \operatorname{Ran} T_{s} \subseteq C_{s} \mathscr{H} \forall t>0, \forall s \in[0, t]$.

ЛЕмма 7. Любое сильно непрерывное коммутативное семейство изометрий $\left\{T_{t}\right\}_{t \geqslant 0}$ такое, что $T_{t} \in \mathscr{E}_{\alpha}(t) \forall t \geqslant 0$, мохнно превратить в сильно непрерывную полугруппу $\left\{\mu(t) T_{t}\right\}_{t \geqslant 0}$ умножением на комплекснозначную непрерывную функиию $\mu(t)$ такую, что $|\mu(t)|=1 \quad \forall t \geqslant 0$.

ДокАЗАТЕЛЬСТво. Поскольку $T_{t+s}, T_{t} T_{s} \in \mathscr{E}_{\alpha}(t+s) \forall t, s \geqslant 0$, то по лемме 2 коммутирующие проекторы $T_{t+s} T_{t+s}^{*}$ и $T_{t} T_{s}\left(T_{t} T_{s}\right)^{*}$ являются минимальными в $\mathscr{R}_{t+s}^{\prime}$. Следовательно, $\operatorname{Ran} T_{t+s}$ и $\operatorname{Ran} T_{t} T_{s}$ либо ортогональны, либо совпадают. Поэтому

$$
F_{s}^{t}=T_{t+s}^{*} T_{t} T_{s}=\left\langle T_{t+s}, T_{t} T_{s}\right\rangle_{\mathscr{E}_{\alpha}(t+s)} I=\lambda(t, s) I
$$

где комплексное число $\lambda(t, s)$ либо равно нулю, либо $|\lambda(t, s)|=1$. При каждом фиксированном $t \geqslant 0$ семейство $\left\{F_{s}^{t}\right\}_{s} \geqslant 0$ сильно непрерывно в силу сильной непрерьвности семейства $\left\{T_{t}\right\}_{t \geqslant 0}$ и непрерывности в сильной операторной топологии операции умножения по совокупности сомножителей на единичном шаре $\mathscr{B}(\mathscr{H})$. Используя топологическую связность $[0 ;+\infty)$, легко показать, что либо $\lambda(t, s)=0 \forall s \geqslant 0$, либо $|\lambda(t, s)|=1$ $\forall s \geqslant 0$. Но $\lambda(t, 0)=1$, так как $F_{0}^{t}=T_{t}^{*} T_{t}=I$. Поэтому имеет место второй случай, а значит, $\operatorname{Ran} T_{t+s}=\operatorname{Ran} T_{t} T_{s}$. С учетом (7) получаем

$$
T_{t+s}=\lambda(t, s) T_{t} T_{s}
$$

где $\lambda(t, s) \in\{z \in \mathbb{C}:|z|=1\}$. Доопределим значения функции $\lambda(t, s)$ для отрицательных значений $t, s$ по формуле $\lambda(-t,-s)=\overline{\lambda(t, s)}, \lambda(-s, t)=\lambda(t,-s)=\lambda(t, s-t)=1$, $s \geqslant t, \lambda(-s, t)=\lambda(t,-s)=\overline{\lambda(s, t-s)}, t \geqslant s, t, s \geqslant 0$ (здесь черта обозначает комплексное сопряжение). Получившаяся функция будет удовлетворять условию 2-коцикла для всех значений аргументов: $\lambda(t+s, r) \lambda(t, s)=\lambda(t, s+r) \lambda(s, r), \lambda(0, t)=\lambda(t, 0)=1$, где $s, t, r \in \mathbb{R}$. В ходе доказательства теоремы 3.2.73 из [4] на с. 302 вводится 2-коцикл $z(t, s)$ со значениями в множестве унитарных операторов, принадлежаших центру произвольной алгебры фон Неймана $\mathscr{M}$. Далее устанавливается сушествование в центре $\mathscr{M}$ семейства $\mu(t)$ со свойством $z(t, s)=\mu(t) \mu(s) \mu(t+s)^{-1}, s, t \in \mathbb{R}$, такого, что для любых фиксированных $\varepsilon>0$ и $\delta>0$, для которых $\|z(t, s)-I\| \leqslant \varepsilon$ при $|t|+|s| \leqslant \delta$, 
выполняется $\|\mu(t)-I\| \leqslant 3 \varepsilon$ при $|t|+|s| \leqslant \delta$. Таким образом, в [4] доказано даже более сильное утверждение, чем нам требуется, и 2-коцикл $\lambda(t, s)$ тривиальный, т.е. $\lambda(t, s)=\mu(t) \mu(s) \mu(t+s)^{-1}$ для некоторой непрерьвной в нуле функции $\mu(t)$. Полагая $S_{t}=\mu(t) T_{t}$, из (8) получаем, что полугруппа изометрий $\left\{S_{t}\right\}_{t \geqslant 0}$ сильно непрерьвна в нуле, а значит, и при всех $t \geqslant 0$, т.е. принадлежит классу $C_{0}$-полугрупп.

Назовем регулярным подрасширением $E_{0}$-полугрупшы эндоморфизмов $\left\{\alpha_{t}\right\}_{t \geqslant 0}$ фактора $\mathscr{M}$ такую $e_{0}$-полугруппу эндоморфизмов $\left\{\varphi_{t}\right\}_{t \geqslant 0}$ фактора $\mathscr{B}(\mathscr{H})$, что при каждом $t>0$ эндоморфизм $\varphi_{t}$ является регулярным подрасширением эндоморфизма $\alpha_{t}$.

В отличие от случая одного регулярного эндоморфизма, существование у регулярной полугруппы хотя бы одного регулярного подрасширения априори не очевидно. Достаточным условием существования регулярного подрасширения у регулярной полугруппы $\left\{\alpha_{t}\right\}_{t \geqslant 0}$ является наличие в $\left\{\mathscr{E}_{\alpha}(t)\right\}_{t \geqslant 0}$ сильно непрерывного коммутативного семейства изометрий $\left\{T_{t}\right\}_{t \geqslant 0}$. Действительно, в силу леммы 7 семейство эндоморфизмов $\left\{T_{t}(\cdot) T_{t}^{*}\right\}_{t \geqslant 0}$ образует $E_{0}$-полугруппу $\mathscr{B}(\mathscr{H})$, причем при каждом $t>0$ эндоморфизм $T_{t}(\cdot) T_{t}^{*}$ является в силу $T_{t} \in \mathscr{E}_{\alpha}(t)$ регулярным подрасширением эндоморфизма $\alpha_{t}$. Следующая теорема 2 (вместе с замечанием 3 ) устанавливает, в частности, что необходимым и достаточным условием существования регулярного подрасширения у полугруппы эндоморфизмов является ее регулярность.

Во множестве всех полугруп эндоморфизмов $\mathscr{B}(\mathscr{H})$ существует естественное отношение частичного порядка: $\left\{\gamma_{t}^{\prime}\right\}_{t \geqslant 0} \succ\left\{\gamma_{t}^{\prime \prime}\right\}_{t \geqslant 0} \Leftrightarrow \gamma_{t}^{\prime}(X) \geqslant \gamma_{t}^{\prime \prime}(X) \forall X \in(\mathscr{B}(\mathscr{H}))_{+}$ $\forall t>0$.

ТЕОРема 2. Множсество $\Phi\left(\left\{\alpha_{t}\right\}\right)$ всех регулярных подрасширений регулярной $E_{0}$-полугруппь $\left\{\alpha_{t}\right\}_{t \geqslant 0}$ фактора $\mathscr{M}$ непусто и содержит наибольший элемент $\left\{\bar{\theta}_{t}\right\}_{t \geqslant 0}$, причем $\bar{\theta}_{t}(\cdot)=\bar{C}_{t} \theta_{t}(\cdot) \forall t \geqslant 0$, где $\bar{C}_{t}=\bigwedge_{r \in[0, t]} C_{r}-$ проектор из $\mathscr{R}_{t}^{\prime}$.

При каждом $t \geqslant 0$ в гильбертовом пространстве $\mathscr{E}_{\alpha}(t)$ существует наибольиее нетривиальное подпространство $\overline{\mathscr{E}}_{\alpha}(t)$ такое, что семейство $\left\{\overline{\mathscr{E}}_{\alpha}(t)\right\}_{t \geqslant 0}$ является СП с операторным умножением в $\mathscr{B}(\mathscr{H})$ в качестве тензорного произведения слоев, причем

$$
\overline{\mathscr{E}}_{\alpha}(t)=\left\{X \in \mathscr{E}_{\alpha}(t): \operatorname{Ran} X \subseteq \bar{C}_{t} \mathscr{H}\right\}
$$

$C \Pi\left\{\overline{\mathscr{E}}_{\alpha}(t)\right\}_{t \geqslant 0}$ ассочиирована по Арвесону с $e_{0}$-полугруппой $\left\{\bar{\theta}_{t}\right\}_{t \geqslant 0}$.

ДоказАтЕльство. Заметим прежде всего, что $\bar{C}_{t}=\bigwedge_{r \in[0, t]} C_{r}-$ ненулевой проектор из $\mathscr{R}_{t}^{\prime}$ при каждом $t>0$ в силу регулярности полугруппы $\left\{\alpha_{t}\right\}_{t \geqslant 0}$ и включений $C_{r} \in \mathscr{R}_{r}^{\prime} \subseteq \mathscr{R}_{t}^{\prime} \forall r \in[0, t]$. Следовательно, $\left\{\bar{\theta}_{t}(\cdot)=\bar{C}_{t} \theta_{t}(\cdot)\right\}_{t \geqslant 0}-$ семейство эндоморфизмов $\mathscr{B}(\mathscr{H})$, причем при каждом $t>0$ эндоморфизм $\bar{\theta}_{t}$ есть регулярное подрасширение эндоморфизма $\alpha_{t}$. Покажем, что

$$
\bar{\theta}_{t}\left(\bar{C}_{s}\right)=\bar{C}_{t+s} \quad \forall t, s>0 .
$$

Пусть $\mathscr{F}_{s}$ - множество всех конечных подмножеств отрезка $[0, s]$, частично упорядо-

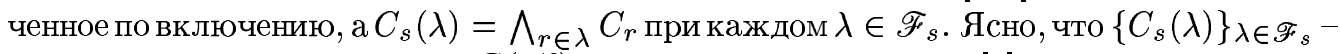
убывающая направленность в $\mathscr{B}(\mathscr{H})_{+}$. В силу леммы 2.4 .19 из [4] существует предел в сильной операторной топологии

$$
s-\lim _{\lambda} C_{s}(\lambda)=\inf _{\lambda} C_{s}(\lambda)=\bar{C}_{s}
$$


Используя лемму 5, получаем

$$
\bar{\theta}_{t}\left(C_{s}(\lambda)\right)=\bar{\theta}_{t}\left(\bigwedge_{r \in \lambda} C_{r}\right)=\bar{\theta}_{t}\left(\prod_{r \in \lambda} C_{r}\right)=\prod_{r \in \lambda} \bar{\theta}_{t}\left(C_{r}\right)=\prod_{r \in \lambda} \bar{C}_{t} \theta_{t}\left(C_{r}\right)=\prod_{r \in \lambda} \bar{C}_{t} C_{t+r} .
$$

Переход к пределу по $\lambda$ с учетом нормальности эндоморфизма $\bar{\theta}_{t}$ дает

$$
\begin{aligned}
\bar{\theta}_{t}\left(\bar{C}_{s}\right) & =\bar{\theta}_{t}\left(C_{s}(\lambda)\right)=s-\lim _{\lambda} \bar{\theta}_{t}\left(C_{s}(\lambda)\right)=s-\lim _{\lambda} \prod_{r \in \lambda} \bar{C}_{t} C_{t+r} \\
& =\inf _{\lambda} \prod_{r \in \lambda} \bar{C}_{t} C_{t+r}=\bar{C}_{t+s} .
\end{aligned}
$$

Таким образом, (9) доказано.

Полугрупповое свойство семейства $\left\{\bar{\theta}_{t}\right\}_{t \geqslant 0}$ легко вьводится из (9) и леммы 6:

$$
\begin{aligned}
\bar{\theta}_{t}\left(\bar{\theta}_{s}(\cdot)\right) & =\bar{\theta}_{t}\left(\bar{C}_{s} \theta_{s}(\cdot)\right)=\bar{\theta}_{t}\left(\bar{C}_{s}\right) \bar{\theta}_{t}\left(\theta_{s}(\cdot)\right)=\bar{C}_{t+s} \bar{C}_{t} \theta_{t}\left(\theta_{s}(\cdot)\right) \\
& =\bar{C}_{t+s} \theta_{t+s}(\cdot)=\bar{\theta}_{t+s}(\cdot)
\end{aligned}
$$

Покажем $\sigma$-слабую непрерьвность полугруппы $\left\{\bar{\theta}_{t}\right\}_{t \geqslant 0}$. Для этого достаточно установить непрерьвность в сильной операторной топологии любой ее траектории

$$
\left\{\bar{\theta}_{t}(X)\right\}_{t \geqslant 0}, \quad X \in \mathscr{B}(\mathscr{H}),
$$

справа при $t=0$. Действительно, сильная непрерывность траектории повлечет ее непрерьвность в $\sigma$-слабой операторной топологии справа при $t=0$, поскольку любая траектория полугрупшы эндоморфизмов лежит в некотором шаре $\mathscr{B}(\mathscr{H})$. Далее, нормальность эндоморфизмов $\bar{\theta}_{t}$ означает существование предсопряженньг операторов ${ }_{*} \bar{\theta}_{t}$ в $\mathscr{B}(\mathscr{H})_{*}$, образующих полугруппу сжатий банахова пространства $\mathscr{B}(\mathscr{H})_{*}$. Из $\sigma$-слабой непрерывности справа при $t=0$ полугрупшы $\left\{\bar{\theta}_{t}\right\}_{t \geqslant 0}$ следует $\sigma\left(\mathscr{B}(\mathscr{H})_{*}\right.$, $\mathscr{B}(\mathscr{H}))$-непрерьвность траекторий полугруппы $\left\{{ }_{*} \bar{\theta}_{t}\right\}_{t \geqslant 0}$ справа при $t=0$. Как известно (см., например, [8, глава 1]), это повлечет непрерывность любой траектории $\left\{{ }_{*} \bar{\theta}_{t}(\omega)\right\}_{t \geqslant 0}, \omega \in \mathscr{B}(\mathscr{H})_{*}$, по норме $\mathscr{B}(\mathscr{H})_{*}$ при всех $t \geqslant 0$. Иначе говоря, $\left\{{ }_{*} \bar{\theta}_{t}\right\}_{t \geqslant 0}$ будет полугруппой класса $C_{0}$ в $\mathscr{B}(\mathscr{H})_{*}$, а сопряженная полугруппа $\left\{\bar{\theta}_{t}\right\}_{t \geqslant 0}$ будет $C_{0}^{*}$-полугруппой в банаховом пространстве $\mathscr{B}(\mathscr{H})$, т.е. $\sigma$-слабо непрерьвной полугруппой эндоморфизмов.

Покажем, что $\exists s-\lim _{t \rightarrow+0} \bar{\theta}_{t}(X)=X \forall X \in \mathscr{B}(\mathscr{H})$. Пусть $\left\{T_{t}\right\}_{t \geqslant 0}$ сильно непрерьвное семейство изометрий, фигурирующее в определении 2 регулярности полугруппы $\left\{\alpha_{t}\right\}_{t \geqslant 0}$. Заметим, что в силу справедливого $\forall \xi \in \mathscr{H}$ соотношения

$$
\left\|T_{t}^{*} \xi-\xi\right\|=\left\|T_{t}^{*} \xi-T_{t}^{*} T_{t} \xi\right\| \leqslant\left\|T_{t}^{*}\right\|\left\|\xi-T_{t} \xi\right\|=\left\|\xi-T_{t} \xi\right\|
$$

существует $s-\lim _{t \rightarrow 0} T_{t}^{*}=I$, а значит, и $s-\lim _{t \rightarrow 0} E_{t}=I$, где $E_{t}=T_{t} T_{t}^{*}$. Из этого замечания и оценки, верной $\forall \xi \in \mathscr{H}, \forall X \in \mathscr{B}(\mathscr{H})$ и $\forall t>0$,

$$
\begin{aligned}
\left\|\bar{\theta}_{t}(X) \xi-X \xi\right\| & =\left\|\bar{\theta}_{t}(X) \xi-\bar{\theta}_{t}(X) E_{t} \xi+\bar{\theta}_{t}(X) E_{t} \xi-X \xi\right\| \\
& \leqslant\left\|\bar{\theta}_{t}(X) \xi-\bar{\theta}_{t}(X) E_{t} \xi\right\|+\left\|\bar{\theta}_{t}(X) E_{t} \xi-X \xi\right\| \\
& \leqslant\left\|\bar{\theta}_{t}(X)\right\|\left\|\xi-E_{t} \xi\right\|+\left\|T_{t} X T_{t}^{*} \xi-X \xi\right\| \\
& =\|X\|\left\|\xi-E_{t} \xi\right\|+\left\|T_{t} X T_{t}^{*} \xi-X \xi\right\|
\end{aligned}
$$


получаем существование указанного вьше предела и, следовательно, $\sigma$-слабую непрерьвность полугруппы $\left\{\bar{\theta}_{t}\right\}_{t \geqslant 0}$.

Пусть $\left\{\varphi_{t}\right\}_{t \geqslant 0}-$ регулярное подрасширение полугруппы $\left\{\alpha_{t}\right\}_{t \geqslant 0}$. Тог да при каждом $r>0$ эндоморфизм $\varphi_{r}$ есть регулярное подрасширение эндоморфизма $\alpha_{r}$ и в силу максимальности $\theta_{r}$ в $\Phi\left(\alpha_{r}\right)$ (теорема 1 ) имеем $\varphi_{r}(I) \leqslant \theta_{r}(I)=C_{r}$. Поэтому при каждом $t>0$ имеем $\varphi_{t}(I) \leqslant \varphi_{r}(I) \leqslant C_{r} \forall r \in[0, t]$. Следовательно,

$$
\varphi_{t}(I) \leqslant \bigwedge_{r \in[0, t]} C_{r}=\bar{C}_{t}=\bar{\theta}_{t}(I) .
$$

Из леммы 4 получаем, что $\varphi_{t} \prec \bar{\theta}_{t} \forall t \geqslant 0$. Таким образом, $\left\{\bar{\theta}_{t}\right\}_{t \geqslant 0}-$ наибольшее регулярное подрасширение полугрупш $\left\{\alpha_{t}\right\}_{t \geqslant 0}$; будем назьвать его регулярным квазирасширением исходной полугруппы.

При каждом $t>0$ множество $\overline{\mathscr{E}}_{\alpha}(t)=\left\{X \in \mathscr{E}_{\alpha}(t): \operatorname{Ran} X \subseteq \bar{C}_{t} \mathscr{H}\right\}$ есть гильбертово пространство операторов, сплетающих эндоморфизм $\bar{\theta}_{t}$. Как показано в [6], $\operatorname{dim} \overline{\mathscr{E}}_{\alpha}(t)$ для всех $t>0$ либо 1 , либо $\infty$. Если $\operatorname{dim} \overline{\mathscr{E}}_{\alpha}(t)=\infty \forall t>0$, то в соответствии с [6] семейство $\left\{\overline{\mathscr{E}}_{\alpha}(t)\right\}_{t \geqslant 0}$ образует СП гильбертовых пространств. Если $\operatorname{dim} \overline{\mathscr{E}}_{\alpha}(t)=1$ $\forall t>0$, то $\bar{\theta}_{t}(\cdot)=T_{t}(\cdot) T_{t}^{*}$ и $\overline{\mathscr{E}}_{\alpha}(t)=\left\{\mathbb{C} T_{t}\right\}$ при каждом $t \geqslant 0$. Лемма 7 показьвает, что семейство $\left\{\overline{\mathscr{E}}_{\alpha}(t)\right\}_{t \geqslant 0}$ в этом случае можно рассматривать как вырожденньй случай CII.

ЗАмЕчАниЕ 3. Утверждение теоремы 2 допускает следующее обращение. Если у $E_{0}$-полугруппы $\left\{\alpha_{t}\right\}_{t \geqslant 0}$ существует хотя бы одно регулярное подрасширение, то такая полугруппа регулярна в смысле определения 2. Действительно, если $\left\{\varphi_{t}\right\}_{t \geqslant 0}-$ такое подрасширение, то с помощью леммы 2.3 в [6] (и рассуждений после нее) нетрудно получить сильно непрерьвное семейство изометрий, сплетающее $\left\{\varphi_{t}\right\}_{t} \geqslant 0$, а значит, удовлетворяющее пункту 1 определения 2 . Из $\varphi_{t}(I) \leqslant \varphi_{s}(I) \leqslant C_{s} \forall t>0, \forall s \in[0, t]$ следует $\bigwedge_{s \leqslant t} C_{s} \geqslant \varphi_{t}(I)>0 \forall t>0$, т.е. выполнение пункта 2 определения 2 .

СледСТВИЕ 1. Семейство $\left\{\mathscr{E}_{\alpha}(t)\right\}_{t \geqslant 0}$ образует СП гильбертовьх пространств с операторным умнохсением в $\mathscr{B}(\mathscr{H})$ в качестве тензорного произведения слоев тогда и только тогда, когда семейство подпространств

$$
\left\{C_{t} \mathscr{H}=\bigvee_{X \in \mathscr{E}_{\alpha}(t)} \operatorname{Ran} X\right\}_{t \geqslant 0}
$$

является монотонно убивающим.

ДокАЗАТЕЛЬСтво. Если $\left\{C_{t}\right\}_{t \geqslant 0}-$ монотонно убывающее семейство проекторов, то $\bar{C}_{t}=C_{t}$, а значит, $\bar{\theta}_{t}=\theta_{t}$ и $\overline{\mathscr{E}}_{\alpha}(t)=\mathscr{E}_{\alpha}(t)$ при каждом $t \geqslant 0$. Обратное утверждение очевидно.

Регулярность некоторых классов полугрупп и конкретные примеры регулярных квазисвободных полугрупп на алгебре КАС рассмотрены в разделе 5 .

4. Коциклические возмущения регулярных полугрупп. Теперь мы исследуем поведение введенных в разделе 3 объектов $\left\{\theta_{t}\right\}_{t \geqslant 0},\left\{\bar{\theta}_{t}\right\}_{t \geqslant 0}\left\{\mathscr{E}_{\alpha}(t)\right\}_{t \geqslant 0}$ и $\left\{\overline{\mathscr{E}}_{\alpha}(t)\right\}_{t \geqslant 0}$ при коциклических возмущениях регулярной полугрупшы $\left\{\alpha_{t}\right\}_{t \geqslant 0}$.

Рассмотрим $E_{0}$-полугруппу $\left\{\alpha_{t}^{u}(\cdot)=U_{t} \alpha_{t}(\cdot) U_{t}^{*}\right\}_{t \geqslant 0}$, где $\left\{U_{t}\right\}_{t \geqslant 0}-$ коцикл в $\mathscr{M}$, т.е. сильно непрерьвное семейство унитарных операторов из $\mathscr{M}$ со свойством $U_{t+s}=$ 
$U_{t} \alpha_{t}\left(U_{s}\right) \forall t, s \geqslant 0$. Полугрупша $\left\{\beta_{t}^{u}=j \circ \alpha_{t}^{u} \circ j^{-1}\right\}_{t \geqslant 0}$ сопряжена с полугрупой $\left\{\beta_{t}=\right.$ $\left.j \circ \alpha_{t} \circ j^{-1}\right\}_{t \geqslant 0}$ посредством коцикла $\left\{U_{t}^{\prime}=J U_{t} J\right\}_{t \geqslant 0}$ :

$$
\beta_{t}^{u}(\cdot)=J \alpha_{t}^{u}(J(\cdot) J) J=J U_{t} \alpha_{t}(J(\cdot) J) U_{t}^{*} J=U_{t}^{\prime} J \alpha_{t}(J(\cdot) J) J U_{t}^{\prime *}=U_{t}^{\prime} \beta_{t}(\cdot) U_{t}^{\prime *} .
$$

Введем сильно непрерывное семейство $\left\{\widetilde{U}_{t}=U_{t} U_{t}^{\prime}\right\}_{t \geqslant 0}$ унитарных операторов в $\mathscr{B}(\mathscr{H})$ и соответствуюшее семейство $\left\{L_{\widetilde{U}}(t)\right\}_{t \geqslant 0}$ преобразований на $\mathscr{B}(\mathscr{H})$ операторного умножения слева: $L_{\widetilde{U}}(t): X \mapsto \widetilde{U}_{t} X \quad \forall X \in \mathscr{B}(\mathscr{H}), \forall t \geqslant 0$.

При каждом $t \geqslant 0$ для любого $T$ из $\mathscr{E}_{\alpha}(t)$ имеем

$$
\begin{aligned}
\widetilde{U}_{t} T A & =U_{t} U_{t}^{\prime} \alpha_{t}(A) T=U_{t} \alpha_{t}(A) U_{t}^{*} U_{t} U_{t}^{\prime} T=\alpha_{t}^{u}(A) \widetilde{U}_{t} T \quad \forall A \in \mathscr{M}, \\
\widetilde{U}_{t} T B & =U_{t} U_{t}^{\prime} \beta_{t}(B) T=U_{t} \beta_{t}(B) U_{t}^{*} U_{t} U_{t}^{\prime} T=\beta_{t}^{u}(B) \widetilde{U}_{t} T \quad \forall B \in \mathscr{M}^{\prime},
\end{aligned}
$$

т.е. $\widetilde{U}_{t} \mathscr{E}_{\alpha}(t) \subseteq \mathscr{E}_{\alpha}^{u}(t)$. Аналогично показывается, что $\widetilde{U}_{t}^{*} \mathscr{E}_{\alpha}^{u}(t) \subseteq \mathscr{E}_{\alpha}(t)$. Следовательно, $\widetilde{U}_{t} \mathscr{E}_{\alpha}(t)=\mathscr{E}_{\alpha}^{u}(t)$. Далее,

$$
\left\langle\widetilde{U}_{t} T_{1}, \widetilde{U}_{t} T_{2}\right\rangle_{\mathscr{E}_{\alpha}^{u}(t)} I=\left(\widetilde{U}_{t} T_{1}\right)^{*} \widetilde{U}_{t} T_{2}=\left(T_{1}\right)^{*} T_{2}=\left\langle T_{1}, T_{2}\right\rangle_{\mathscr{E}_{\alpha}(t)} I \quad \forall T_{1}, T_{2} \in \mathscr{E}_{\alpha}(t) .
$$

Таким образом, $L_{\widetilde{U}}(t)$ есть унитарное преобразование из $\mathscr{E}_{\alpha}(t)$ на $\mathscr{E}_{\alpha}^{u}(t)$ при каждом $t \geqslant 0$.

Пусть $\left\{\varphi_{t}\right\}_{t \geqslant 0}$ - какое-либо регулярное подрасширение полугрушы $\left\{\alpha_{t}\right\}_{t \geqslant 0}$. Рассмотрим семейство эндоморфизмов $\left\{\varphi_{t}^{u}(\cdot)=\widetilde{U}_{t} \varphi_{t}(\cdot) \widetilde{U}_{t}^{*}\right\}_{t \geqslant 0}$. При каждом $t>0$ имеем

$$
\begin{array}{rlr}
\varphi_{t}^{u}(A) & =\widetilde{U}_{t} \varphi_{t}(A) \widetilde{U}_{t}^{*}=\widetilde{U}_{t} \varphi_{t}(I) \alpha_{t}(A) \widetilde{U}_{t}^{*} \\
& =\widetilde{U}_{t} \varphi_{t}(I) \widetilde{U}_{t}^{*} \widetilde{U}_{t} \alpha_{t}(A) \widetilde{U}_{t}^{*}=\varphi_{t}^{u}(I) \alpha_{t}^{u}(A) & \\
\varphi_{t}^{u}(B) & =\widetilde{U}_{t} \varphi_{t}(B) \widetilde{U}_{t}^{*}=\widetilde{U}_{t} \varphi_{t}(I) \alpha_{t}(B) \widetilde{U}_{t}^{*} & \\
& =\widetilde{U}_{t} \varphi_{t}(I) \widetilde{U}_{t}^{*} \widetilde{U}_{t} \beta_{t}(B) \widetilde{U}_{t}^{*}=\varphi_{t}^{u}(I) \beta_{t}^{u}(B) \quad \forall B \in \mathscr{M}^{\prime},
\end{array}
$$

т.е. $\varphi_{t}^{u}$ - регулярное подрасширение эндоморфизма $\varphi_{t}$. Далее заметим, что для всех $t, s>0$ в силу леммы 3

$$
\widetilde{U}_{t} \varphi_{t}\left(\widetilde{U}_{s}\right)=U_{t} U_{t}^{\prime} \varphi_{t}(I) \alpha_{t}\left(U_{t}\right) \beta_{t}\left(U_{t}^{\prime}\right)=U_{t+s} U_{t+s}^{\prime} \varphi_{t}(I)
$$

и, следовательно,

$$
\begin{aligned}
\varphi_{t}^{u}\left(\varphi_{s}^{u}(\cdot)\right) & =\widetilde{U}_{t} \varphi_{t}\left(\widetilde{U}_{s} \varphi_{s}(\cdot) \widetilde{U}_{s}^{*}\right) \widetilde{U}_{t}^{*}=\widetilde{U}_{t} \varphi_{t}\left(\widetilde{U}_{s}\right) \varphi_{t}\left(\varphi_{s}(\cdot)\right) \varphi_{t}\left(\widetilde{U}_{s}^{*}\right) \widetilde{U}_{t}^{*} \\
& =\widetilde{U}_{t+s} \varphi_{t+s}(\cdot) \widetilde{U}_{t+s}^{*}=\varphi_{t+s}^{u}(\cdot),
\end{aligned}
$$

т.е. сильно непрерьвное семейство эндоморфизмов $\left\{\varphi_{t}^{u}(\cdot)\right\}_{t \geqslant 0}$ образует $e_{0}$-полугрупу. Учитьвая (10), заключаем, что $\left\{\varphi_{t}^{u}(\cdot)\right\}_{t \geqslant 0}$ - регулярное подрасширение $E_{0}$-полугруппы $\left\{\alpha_{t}^{u}\right\}_{t \geqslant 0}$. В силу замечания 3 полугруппа $\left\{\alpha_{t}^{u}\right\}_{t \geqslant 0}$ является регулярной. Следовательно, для этой полугруппы можно ввести семейство эндоморфизмов $\left\{\theta_{t}^{u}\right\}_{t \geqslant 0}, E_{0}$-полугруппу $\left\{\bar{\theta}_{t}^{u}\right\}_{t \geqslant 0}$ и СП $\left\{\overline{\mathscr{E}}_{\alpha}^{u}(t)\right\}_{t \geqslant 0}$.

Если $\left\{T_{t}^{i}\right\}_{i=1}^{i=n}-$ ОНБ в $\mathscr{E}_{\alpha}(t)$, то по доказанному выше $\left\{\widetilde{U}_{t} T_{t}^{i}\right\}_{i=1}^{i=n}$ является ОНБ в $\mathscr{E}_{\alpha}^{u}(t)$. Поэтому в силу теоремы 1 при всех $t \geqslant 0$

$$
\theta_{t}^{u}(\cdot)=\sum_{i=1}^{n} \widetilde{U}_{t} T_{i}(\cdot)\left(\widetilde{U}_{t} T_{i}\right)^{*}=\widetilde{U}_{t}\left(\sum_{i=1}^{n} T_{i}(\cdot) T_{i}^{*}\right) \widetilde{U}_{t}^{*}=\widetilde{U}_{t} \theta_{t}(\cdot) \widetilde{U}_{t}^{*} .
$$


В частности,

$$
C_{t}^{u}=\widetilde{U}_{t} C_{t} \widetilde{U}_{t}^{*}
$$

Аналог соотношения (11) для эндоморфизмов полугруппы $\left\{\bar{\theta}_{t}\right\}_{t \geqslant 0}$ будет установлен, если доказать для семейства $\left\{\bar{C}_{t}\right\}_{t \geqslant 0}$ аналогичное (12) равенство

$$
\bar{C}_{t}^{u}=\widetilde{U}_{t} \bar{C}_{t} \widetilde{U}_{t}^{*}
$$

При каждом $t>0$ для всех $r \in[0, t]$ в силу $C_{r} \in \mathscr{R}_{r} \wedge \mathscr{R}_{r}^{\prime}$ и (12) имеем

$$
\begin{aligned}
\widetilde{U}_{t} C_{r} \widetilde{U}_{t}^{*} & =U_{r} \alpha_{r}\left(U_{t-r}\right) U_{r}^{\prime} \beta_{r}\left(U_{t-r}^{\prime}\right) C_{r}\left(U_{r} \alpha_{r}\left(U_{t-r}\right) U_{r}^{\prime} \beta_{r}\left(U_{t-r}^{\prime}\right)\right)^{*} \\
& =U_{r} U_{r}^{\prime} C_{r} U_{r}^{\prime *} U_{r}^{*}=\widetilde{U}_{r} C_{r} \widetilde{U}_{r}^{*}=C_{r}^{u}
\end{aligned}
$$

Пусть $\mathscr{F}_{t}$ - множество всех конечных подмножеств отрезка $[0, t]$, частично упорядоченное по включению, а $C_{t}(\lambda)$ и $C_{t}^{u}(\lambda)$ при каждом $\lambda \in \mathscr{F}_{t}$ есть точные нижние грани наборов проекторов $\left\{C_{r}\right\}_{r \in \lambda}$ и $\left\{C_{r}^{u}\right\}_{r \in \lambda}$ соответственно. Тогда из коммутативности этих наборов и последнего равенства следует, что

$$
\widetilde{U}_{t} C_{t}(\lambda) \widetilde{U}_{t}^{*}=\widetilde{U}_{t}\left(\prod_{r \in \lambda} C_{r}\right) \widetilde{U}_{t}^{*}=\left(\prod_{r \in \lambda} C_{r}^{u}\right)=C_{t}^{u}(\lambda) .
$$

Поскольку проекторы $\bar{C}_{t}$ и $\bar{C}_{t}^{u}$ есть $\sigma$-слабые пределы направленностей $\left\{C_{t}(\lambda)\right\}_{\lambda \in \mathscr{F}_{t}}$

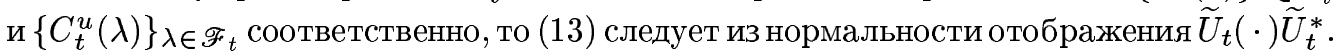

Из (11) и (13) следует, что

$$
\bar{\theta}_{t}^{u}(\cdot)=C_{t}^{u} \theta_{t}^{u}(\cdot)=\widetilde{U}_{t} C_{t}(\cdot) \theta_{t}(\cdot) \widetilde{U}_{t}^{*}=\widetilde{U}_{t} \bar{\theta}_{t}(\cdot) \widetilde{U}_{t}^{*}
$$

Покажем изоморфность СП $\left\{\overline{\mathscr{E}}_{\alpha}(t)\right\}_{t \geqslant 0}$ и $\left\{\overline{\mathscr{E}}_{\alpha}^{u}(t)\right\}_{t \geqslant 0}$. Заметим, что если полугруппа $\left\{\bar{\theta}_{t}\right\}_{t \geqslant 0}$ унитальна, то семейство $\left\{\widetilde{U}_{t}\right\}_{t \geqslant 0}$ есть коцикл в $\mathscr{B}(\mathscr{H})$ и $(11)$ означает коциклическую сопряженность полугрупп $\left\{\bar{\theta}_{t}=\theta_{t}\right\}_{t \geqslant 0}$ и $\left\{\bar{\theta}_{t}^{u}=\theta_{t}^{u}\right\}_{t \geqslant 0}$, а значит, и изоморфность СП $\left\{\overline{\mathscr{E}}_{\alpha}(t)=\mathscr{E}_{\alpha}(t)\right\}_{t \geqslant 0}$ и $\left\{\overline{\mathscr{E}}_{\alpha}^{u}(t)=\mathscr{E}_{\alpha}^{u}(t)\right\}_{t \geqslant 0}$ в силу теоремы 3.8.1 из [6].

В случае неунитальности $\left\{\theta_{t}\right\}_{t \geqslant 0}$ семейство $\left\{\widetilde{U}_{t}\right\}_{t \geqslant 0}$ не является коциклом. Тем не менее, можно непосредственно показать изоморфность СП $\left\{\overline{\mathscr{E}}_{\alpha}(t)\right\}_{t \geqslant 0}$ и $\left\{\overline{\mathscr{E}}_{\alpha}^{u}(t)\right\}_{t \geqslant 0}$, обобщая доказательство теоремы 3.8.1 из [6]. В силу (13) и (14) все необходимые изменения в указанном доказательстве касаются проверки мультипликативного свойства отображения

$$
\Psi:(t, T) \mapsto\left(t, \widetilde{U}_{t} T\right) \quad \forall T \in \overline{\mathscr{E}}_{\alpha}(t)
$$

из $\left\{\overline{\mathscr{E}}_{\alpha}(t)\right\}_{t \geqslant 0 \text { на }}\left\{\overline{\mathscr{E}}_{\alpha}^{u}(t)\right\}_{t \geqslant 0}$.

Пусть $T \in \overline{\mathscr{E}}_{\alpha}(t)$ и $S \in \overline{\mathscr{E}}_{\alpha}(s)$. Тогда, учитьвая лемму 3 , теорему 1 и равенство $T=T I=\theta_{t}(I) T=C_{t} T$, имеем

$$
\begin{aligned}
\widetilde{U}_{t} T \widetilde{U}_{s} S & =\widetilde{U}_{t} \theta_{t}\left(\widetilde{U}_{s}\right) T S=U_{t} U_{t}^{\prime} \theta_{t}\left(U_{s} U_{s}^{\prime}\right) T S=U_{t} U_{t}^{\prime} \alpha_{t}\left(U_{s}\right) \beta_{t}\left(U_{s}^{\prime}\right) C_{t} T S \\
& =U_{t+s} U_{t+s}^{\prime} T S=\widetilde{U}_{t+s} T S .
\end{aligned}
$$

Следовательно, отображение $\Psi$ сохраняет "послойное" умножение. Это завершает обобщение доказательства теоремы 3.8.1 из [6] на случай СП $\left\{\overline{\mathscr{E}}_{\alpha}(t)\right\}_{t \geqslant 0}$ и $\left\{\overline{\mathscr{E}}_{\alpha}^{u}(t)\right\}_{t \geqslant 0}$. Таким образом, установлена следующая 
ТЕОрема 3. Пусть $\left\{\alpha_{t}\right\}_{t \geqslant 0}-$ регулярная полугруппа $u\left\{\alpha_{t}^{u}(\cdot)=U_{t} \alpha_{t}(\cdot) U_{t}^{*}\right\}_{t \geqslant 0}$, где $\left\{U_{t}\right\}_{t \geqslant 0}-$ коцикл в $\mathscr{M}$. Тогда возмущенная полугруппа $\left\{\alpha_{t}^{u}\right\}_{t \geqslant 0}$ также регулярна. Сильно непрерывное семейство $\left\{\widetilde{U}_{t}=U_{t} U_{t}^{\prime}=U_{t} J U_{t} J\right\}_{t \geqslant 0}$ унитарных операторов порождает семейство $\left\{L_{\widetilde{U}}(t)\right\}_{t \geqslant 0}$ унитарных преобразований из $\mathscr{E}_{\alpha}(t)$ на $\mathscr{E}_{\alpha}(t)$ и связывает регулярные квазирасширения:

$$
\theta_{t}^{u}(\cdot)=\widetilde{U}_{t} \theta_{t}(\cdot) \widetilde{U}_{t}^{*}, \quad \bar{\theta}_{t}^{u}(\cdot)=\widetilde{U}_{t} \bar{\theta}_{t}(\cdot) \widetilde{U}_{t}^{*} .
$$

СП гильбертовых пространств $\left\{\overline{\mathscr{E}}_{\alpha}(t)\right\}_{t \geqslant 0} u\left\{\overline{\mathscr{E}}_{\alpha}^{u}(t)\right\}_{t \geqslant 0}$ изоморфны.

Последнее утверждение теоремы 3 показьвает, что СП $\left\{\overline{\mathscr{E}}_{\alpha}(t)\right\}_{t \geqslant 0}$ обладает свойством устойчивости по отношению к коциклическим возмущениям полугрупшы $\left\{\alpha_{t}\right\}_{t \geqslant 0}$. Это обосновьвает следующее

ОПРЕДЕЛЕНИЕ 3. СП $\left\{\overline{\mathscr{E}}_{\alpha}(t)\right\}_{t \geqslant 0}$ будем назьвать системой-произведением, ассоииированной с $E_{0}$-nолугруппой $\left\{\alpha_{t}\right\}_{t \geqslant 0}$.

Такое определение согласуется с данньп в [3] для квазисвободной $E_{0}$-полугрупшы.

ЗАмЕЧАниЕ 4. Пусть $\left\{\theta_{t}\right\}_{t \geqslant 0}-$ регулярное квазирасширение полугруппы $\left\{\alpha_{t}\right\}_{t \geqslant 0}$. Рассмотрим $\alpha$-коцикл $\left\{U_{t}\right\}_{t \geqslant 0}$ в $\mathscr{M}$ и $\beta$-коцикл $\left\{U_{t}^{\prime}=J U_{t} J\right\}_{t \geqslant 0}$ в $\mathscr{M}^{\prime}$. Рассуждения, аналогичные приведенным вьше в данном разделе, показывают, что формула

$$
\theta_{t}^{u}(\cdot)=U_{t}^{\prime} \theta_{t}(\cdot) U_{t}^{\prime *}, \quad t \geqslant 0
$$

корректно задает некоторое (нерегулярное) квазирасширение $\left\{\theta_{t}^{u}\right\}_{t \geqslant 0}$ полугрупшы $\left\{\alpha_{t}\right\}_{t \geqslant 0}$. В следующем разделе показано, что в случае квазисвободных полугрупा на факторах, полученных из представлений алгебры КАС, это утверждение допускает обращение: две $E_{0}$-полугруппы $\left\{\alpha_{t}\right\}_{t \geqslant 0}$ и $\left\{\gamma_{t}\right\}_{t \geqslant 0}$ на факторах $\mathscr{M}$ и $\mathscr{M}^{\prime}$ соответственно имеют общее расширение на $\mathscr{B}(\mathscr{H})$ только тогда, когда полугруппа $\left\{j^{-1} \circ \gamma_{t} \circ j\right\}_{t \geqslant 0}$ коциклически сопряжена с полугруппй $\left\{\alpha_{t}\right\}_{t \geqslant 0}$. Это обстоятельство оправдывает выделение регулярных расширений среди других возможных расширений $E_{0}$-полугрупшы $\left\{\alpha_{t}\right\}_{t \geqslant 0}$ до полугрупшы на $\mathscr{B}(\mathscr{H})$.

5. Примеры регулярных полугрупп. Прежде всего отметим, что любая $E_{0}$-полугруппа $\left\{\alpha_{t}\right\}_{t \geqslant 0}$ на факторе $\mathscr{M}$ типа I является регулярной, поскольку у такой полугруппы всегда есть регулярное расширение $\left\{\theta_{t}\right\}_{t \geqslant 0}$ (замечание 3). Действительно, в этом случае существует изоморфизм между $\mathscr{B}(\mathscr{H})$ и $\mathscr{M} \bar{\otimes} \mathscr{M}^{\prime}[5]$, и регулярное расширение $\theta_{t}$ соответствует $E_{0}$-полугрупше $\alpha_{t} \otimes \beta_{t}$ при этом изоморфизме.

Покажем регулярность важного класса полугрупп на факторах любого типа. В соответствии с [9] полугрупша $\left\{\alpha_{t}\right\}_{t \geqslant 0}$ на факторе $\mathscr{M}$ называется вполне совместимой с точным нормальным состоянием $\omega(\cdot)=(\Omega,(\cdot) \Omega)$, если $\omega \circ \alpha_{t}=\omega$ и $\alpha_{t} \circ \sigma_{s}^{\omega}=\sigma_{s}^{\omega} \circ \alpha_{t}$ $\forall t, s \geqslant 0$, где $\sigma_{s}^{\omega}$ - модулярная группа, ассоциированная с состоянием $\omega$. Свойство совместимости $\omega \circ \alpha_{t}=\omega$ означает, что соответствие $T_{t}: A \Omega \mapsto \alpha_{t}(A) \Omega \forall A \in \mathscr{M}, \forall t \geqslant 0$ корректно определяет сильно непрерывную полугрупп изометрий $\left\{T_{t}\right\}_{t \geqslant 0}$ на $\mathscr{H}$ такую, что $T_{t} A=\alpha_{t}(A) T_{t} \forall A \in \mathscr{M}$.

Свойство $\alpha_{t} \circ \sigma_{s}^{\omega}=\sigma_{s}^{\omega} \circ \alpha_{t} \forall t, s \geqslant 0$ показьвает (см. [10]), что полугрупша $\left\{T_{t}\right\}_{t \geqslant 0}$ коммутирует с модулярной инволюцией $J$, ассоциированной с вектором $\Omega$. Следовательно, при каждом $t \geqslant 0$

$T_{t} B=T_{t} J J B J J=J T_{t} J B J J=J \alpha_{t}(J B J) T_{t} J=J \alpha_{t}(J B J) J T_{t}=\beta_{t}(B) T_{t} \quad \forall B \in \mathscr{M}^{\prime}$. 
Таким образом, $T_{t} \in \mathscr{E}_{\alpha}(t) \forall t \geqslant 0$ и полугруппа $\left\{\alpha_{t}\right\}_{t \geqslant 0}$ регулярна в силу замечания 2 к определению 2.

Рассмотрим $C^{*}$-алгебру канонических антикоммутационных соотношений $\mathscr{A}(\mathscr{K})$ над сепарабельньм гильбертовым пространством $\mathscr{K}$. Образуюшими $\mathscr{A}(\mathscr{K})$ являются операторы рождения и уничтожения $a^{*}(f)$ и $a(g)$, где $f, g \in \mathscr{K}$, в антисимметричном пространстве Фока $\mathscr{F}(\mathscr{K})$ с вакуумньм вектором $\Omega$ (см. [11]). Фиксируем в $\mathscr{K}$ положительный оператор $R, 0<R<I, \operatorname{ker} R=\operatorname{ker}(I-R)=0$, антиунитарньй оператор $\mathscr{J}$ и построим представления $\pi$ алгебры $\mathscr{A}(\mathscr{K} \oplus \mathscr{K})$ и $\pi_{R}$ алгебры $\mathscr{A}(\mathscr{K})$ в гильбертовом пространстве $\mathscr{H}=\mathscr{F}(\mathscr{K}) \otimes \mathscr{F}(\mathscr{K})$ по формуле

$$
\begin{gathered}
\pi(a(f \oplus 0))=\pi_{R}(a(f))=a\left((1-R)^{1 / 2} f\right) \otimes \Gamma+1 \otimes a\left(R^{1 / 2} \mathscr{J} f\right), \\
\pi(a(0 \oplus f))=a\left(R^{1 / 2} f\right) \otimes \Gamma-1 \otimes a\left((1-R)^{1 / 2} \mathscr{J} f\right),
\end{gathered}
$$

$f \in \mathscr{K}$. Здесь $\Gamma$ - унитарный оператор, полностью определенный формулой $Г а(f)=$ $-a(f) \Gamma, \Gamma \Omega=\Omega, f \in \mathscr{K}$. Определим гиперфинитные факторы $\mathscr{M}_{R}=\pi_{R}(\mathscr{A}(\mathscr{K}))^{\prime \prime}$ и $\mathscr{M}_{P}=\pi(\mathscr{A}(\mathscr{K} \oplus \mathscr{K}))^{\prime \prime}=\mathscr{B}(\mathscr{H})$ и рассмотрим два векторных состояния на них:

$$
\begin{array}{ll}
\omega_{R}(x)=\left\langle\Omega \otimes \Omega, \pi_{R}(x) \Omega \otimes \Omega\right\rangle, & x \in \mathscr{A}(\mathscr{K} \oplus 0), \\
\omega_{P}(x)=\langle\Omega \otimes \Omega, \pi(x) \Omega \otimes \Omega\rangle, & x \in \mathscr{A}(\mathscr{K} \oplus \mathscr{K}) .
\end{array}
$$

Заметим, что

$$
\begin{array}{ll}
\omega_{R}\left(\pi\left(a^{*}(f) a(g)\right)\right)=(f, R g), & f, g \in \mathscr{K}, \\
\omega_{P}\left(\pi\left(a^{*}(f) a(g)\right)\right)=(f, P g), & f, g \in \mathscr{K} \oplus \mathscr{K},
\end{array}
$$

где

$$
P=\left(\begin{array}{cc}
R & R^{1 / 2}(I-R)^{1 / 2} \\
R^{1 / 2}(I-R)^{1 / 2} & I-R
\end{array}\right)
$$

- ортогональный проектор в гильбертовом пространстве $\mathscr{K} \oplus \mathscr{K}$. Состояние $\omega_{R}$ точное, а состояние $\omega_{P}$ чистое и получается в ходе процедуры “очищения" [12] из $\omega_{R}$. Операторы

$$
b(f)=\Gamma \otimes \Gamma \pi(a(0 \oplus f)), \quad b^{*}(f)=\pi\left(a^{*}(0 \oplus f)\right) \Gamma \otimes \Gamma, \quad f \in \mathscr{K},
$$

порождают коммутант $\mathscr{M}_{R}^{\prime}$, причем $b(f)=J \pi_{R}(a(f)) J, f \in \mathscr{K}$, где $J$ есть модулярная инволюция на $\mathscr{M}_{R}$, отвечающая точному нормальному состоянию $\omega_{R}$. Пусть $V$ и $W$ - изометрические операторы в $\mathscr{K}$, коммутирующие с $R$. Рассмотрим квазисвободные эндоморфизмы $\alpha$ фактора $\mathscr{M}_{R}$ и $\gamma$ коммутанта $\mathscr{M}_{R}^{\prime}$, полученные подъемом $V$ и $W$ : $\alpha\left(\pi_{R}(a(f))\right)=\pi_{R}(a(V f)), \gamma(b(f))=b(W f), f \in \mathscr{K}$. Предположим, что у операторов $V$ и $W$ существуют минимальные унитарные дилатации $V^{\prime}$ и $W^{\prime}$, действующие на одном гильбертовом пространстве $\mathscr{K}^{\prime}, \mathscr{K} \subset \mathscr{K}^{\prime}$, и найдется положительное сжатие $R^{\prime}$ в $\mathscr{K}^{\prime}$ такое, что $V^{\prime} R=R^{\prime} V, W^{\prime} R=R^{\prime} W$ (о существовании такого $R^{\prime}$ см. в [13]). Зададим квазисвободный эндоморфизм $\theta C^{*}$-алгебры, порожденной $\mathscr{M}_{R}$ и $\mathscr{M}_{R}^{\prime}$, полагая $\left.\theta\right|_{\mathscr{M}_{R}}=\alpha,\left.\theta\right|_{\mathscr{M}_{R}^{\prime}}=\gamma$. Для такого $\theta$ (заданного квазисвободным подъемом изометрических операторов $V$ и $W$ ) верна

Теорема 4. Эндоморфизм $\theta$ продолжается на $\mathscr{B}(\mathscr{H})$ тогда и только тогда, когда выполнено условие $R^{\prime 1 / 2}\left(I-R^{\prime}\right)^{1 / 2}\left(V^{\prime}-W^{\prime}\right) \in s_{2}$ (где $s_{2}$ - класс операторов Гильберта-Шмидта в $\left.\mathscr{K}^{\prime}\right)$. 
ДокАЗАТЕЛЬСТво. Как доказано Араки в [14], любой квазисвободньй *-автоморфизм $\theta^{\prime}$, заданный на $C^{*}$-алгебре $\pi\left(A\left(\mathscr{K}^{\prime} \oplus \mathscr{K}^{\prime}\right)\right)$ условием

$$
\theta^{\prime}(\pi(a(f \oplus g)))=\pi\left(a\left(V^{\prime} f \oplus W^{\prime} g\right)\right), \quad f, g \in \mathscr{K}^{\prime}
$$

будет продолжаться на фактор $\mathscr{M}_{P^{\prime}}$, отвечающий проектору

$$
P^{\prime}=\left(\begin{array}{cc}
R^{\prime} & R^{\prime 1 / 2}\left(I-R^{\prime}\right)^{1 / 2} \\
R^{\prime 1 / 2}\left(I-R^{\prime}\right)^{1 / 2} & I-R^{\prime}
\end{array}\right)
$$

тогда и только тогда, когда

$$
\left(\begin{array}{cc}
V^{\prime} & 0 \\
0 & W^{\prime}
\end{array}\right) P^{\prime}-P^{\prime}\left(\begin{array}{cc}
V^{\prime} & 0 \\
0 & W^{\prime}
\end{array}\right) \in s_{2}
$$

Унитарньй оператор

$$
\left(\begin{array}{cc}
V^{\prime} & 0 \\
0 & W^{\prime}
\end{array}\right)
$$

в пространстве $\mathscr{K}^{\prime} \oplus \mathscr{K}^{\prime}$ удовлетворяет этому условию и корректно определяет автоморфизм $\theta^{\prime}$. Автоморфизм $\theta^{\prime}$ обладает свойством $\theta^{\prime}(\Gamma \otimes \Gamma)=\Gamma \otimes \Gamma$, так что $\theta^{\prime}(b(f))=$ $b\left(W^{\prime} f\right), f \in \mathscr{K}^{\prime}$. Фактор $\mathscr{M}_{P}=\mathscr{B}(\mathscr{H}) \subset \mathscr{M}_{P^{\prime}}$ является инвариантным относительно действия $\theta^{\prime}$. Рассматривая сужение, получаем $\theta^{\prime} \mid \mathscr{M}_{P}=\theta$. Отметим, что в случае $V=W$ эндоморфизм $\theta$ является регулярным расширением $\alpha$.

ЗАмЕчАниЕ 5. По поводу условия предыдущей теоремы см. также [15].

Пусть теперь $\left\{V_{t}\right\}_{t \geqslant 0}-C_{0}$-полугруппа изометрических операторов в гильбертовом пространстве $\mathscr{K}$. Тогда семейство $\left\{\alpha_{t}\right\}_{t \geqslant 0}$ квазисвободных эндоморфизмов фактора $\mathscr{M}_{R}$, задаваемых при каждом $t \geqslant 0$ формулой $\alpha_{t}\left(\pi_{R}(a(f))\right)=\pi_{R}\left(a\left(V_{t} f\right)\right), f \in \mathscr{K}$, является $E_{0}$-полугруппой. Введем расширяющееся семейство гильбертовых пространств $\left\{\mathscr{H}_{t}\right\}_{t \geqslant 0}$, вложенных в пространство $\mathscr{F}(\mathscr{K})$, где при каждом $t \geqslant 0$ пространство $\mathscr{H}_{t}$ порождается всевозможными векторами $a^{\#}\left(f_{1}\right) a^{\#}\left(f_{2}\right) \cdots a^{\#}\left(f_{n}\right) \Omega, f_{i} \in \operatorname{ker} V_{t}^{*}, 1 \leqslant$ $i \leqslant n$; здесь $a^{\#}=a^{*}$ или $a$. Семейство $\left\{\mathscr{H}_{t}\right\}_{t \geqslant 0}$ является СП гильбертовых пространств [3]. Введем СП $\left\{\mathscr{K}_{t}=\mathscr{H}_{t} \otimes \mathscr{J} \mathscr{H}_{t}\right\}_{t \geqslant 0}$. Пусть $\left\{\theta_{t}\right\}_{t \geqslant 0}$ есть регулярное расширение $\left\{\alpha_{t}\right\}_{t \geqslant 0}$ (построенное в [3]), а $P_{\Omega}$ - одномерньй проектор на вектор $\Omega \otimes \Omega$; тогда

$$
\mathscr{K}_{t}=\theta_{t}\left(P_{\Omega}\right) \mathscr{H}, \quad t \geqslant 0 .
$$

Таким образом, СП, ассоциированная с регулярным расширением, получается “удваиванием" СП, ассоциированной с исходной полугруппой. Нетрудно видеть (см. [3]), что семейство $\left\{\mathscr{K}_{t}\right\}_{t \geqslant 0}$ монотонно возрастает. В общем случае для $e_{0}$-полугрупшы $\left\{\gamma_{t}\right\}_{t \geqslant 0}$ эндоморфизмов $\mathscr{B}(\mathscr{H})$ семейство $\left\{\mathscr{K}_{t}=\gamma_{t}(P) \mathscr{H}\right\}_{t \geqslant 0}$, где $P$ - одномерньй проектор, задает СП [7]. Однако в общем случае семейство $\left\{\mathscr{K}_{t}\right\}_{t \geqslant 0}$ не обязано быть монотонно возрастающим. Монотонное возрастание имеет место в случае совместимости полугруппы $\left\{\gamma_{t}\right\}_{t \geqslant 0}$ с векторным состоянием. 
ЗАмЕчАниЕ 6. В отличие от [3], рассмотрим две $E_{0}$-полугрупшы $\left\{\alpha_{t}\right\}_{t \geqslant 0}$ и $\left\{\gamma_{t}\right\}_{t \geqslant 0}$ на факторе $\mathscr{M}_{R}$, определенные соответственно квазисвободным подъемом $C_{0}$-полугрупп $\left\{V_{t}\right\}_{t \geqslant 0}$ и $\left\{W_{t}\right\}_{t \geqslant 0}$ в пространстве $\mathscr{K}$. Предположим, что у полугрупा $\left\{V_{t}\right\}_{t \geqslant 0}$ и $\left\{W_{t}\right\}_{t \geqslant 0}$ существуют минимальные унитарные дилатации $\left\{V_{t}^{\prime}\right\}_{t \geqslant 0}$ и $\left\{W_{t}^{\prime}\right\}_{t \geqslant 0}$, действующие на одном гильбертовом пространстве $\mathscr{K}^{\prime}, \mathscr{K} \subset \mathscr{K}^{\prime}$, и найдется положительное сжатие $R^{\prime}$ в $\mathscr{K}^{\prime}$ такое, что $V_{t}^{\prime} R=R^{\prime} V_{t}, W_{t}^{\prime} R=R^{\prime} W_{t} \forall t \geqslant 0$. Условие

$$
R^{\prime 1 / 2}\left(I-R^{\prime}\right)^{1 / 2}\left(V_{t}^{\prime}-W_{t}^{\prime}\right) \in s_{2}, \quad t>0
$$

достаточно для коциклической сопряженности $\left\{\alpha_{t}\right\}_{t \geqslant 0}$ и $\left\{\gamma_{t}\right\}_{t \geqslant 0}$ (см. [16]). Таким образом, класс всех расширений на коммутант, упомянутых в замечании 4, описьвается в квазисвободном случае полугрупповым аналогом теоремы 4.

\section{СПИСОК ЦИТИРОВАННОЙ ЛИТЕРАТУРЫ}

[1] Powers R. T. Possible classification of continuous spatial semigroups of $*$-endomorfisms of $\mathscr{B}(\mathscr{H}) / /$ Proceedings of Symposia in Pure Math. 1996. V. 59. P. 161-173.

[2] Powers R. T. Recent results concerning $E_{0}$-semigroup of $\mathscr{B}(\mathscr{H}) / /$ Operator Algebras and Quantum Field Theory / ed. S. Doplicher et al. Proc. of Conf. Dedicated to D. Kastler. Cambridge (M.A.): Int. Press, 1997. P. 515-524.

[3] Амосов Г. Г., Булинский А. В. Индекс Пауэрса-Арвесона для квазисвободных динамических полугрупп // Матем. заметки. 1997. Т. 62. №6. С. 933-936.

[4] Браттели У., Робинсон Д. Операторные алгебры и квантовая статистическая механика I. М.: Мир, 1982.

[5] Kadison R., Ringrose J. Fundamentals of the Theory of Operator Algebras. I. London-New York: Acad. Press, 1983; II. London-New York: Acad. Press, 1986.

[6] Arveson W. Continuous analogues of Fock space // Mem. Amer. Math. Soc. 1989. V. 409. P. 1-66.

[7] Bhat B. V.R. An index theory for quantum dynamical semigroup // Trans. Amer. Math. Soc. 1996. V. 348. P. 561-583.

[8] Davies E. B. One-Parameter Semigroups. London-New York: Acad. Press, 1980.

[9] Булинский А. В. Алгебраические $K$-системы и полупотоки сдвигов Пауэрса // УМН. 1996. T. 52. № 5. C. $145-146$.

[10] Accardi L., Cecchini C. Conditional expectation in von Neumann algebras and a theorem of Takesaki // J. Funct. Anal. 1982. V. 45. P. 245-273.

[11] Bratteli O., Robinson D. Operator Algebras and Quantum Statistical Mechanics II. New York: Springer-Verlag, 1981.

[12] Powers R. T., Stormer E. Free states of canonical anticommutation relations // Commun. Math. Phys. 1970. V. 16. P. 1-33.

[13] Evans D. Completely positive quasifree maps on the CAR algebra // Commun. Math. Phys. 1979. V. 70. P. 53-68.

[14] Araki H. On quasifree states of CAR and Bogoliubov automorphisms // Publ. RIMS (Kyoto Univ. ). 1971. V. 6. P. 385-442.

[15] Amosov G. G. Cocycle perturbation of quasifree algebraic $K$-flow leads to the required asymptotic dynamics of the associated completely positive semigroup // Infinit. Dimen. Anal. Quant. Prob. Rel. Topics. 2000. V. 2. № 3. P. 237-246.

[16] Murakami T., Yamagami S. On types of quasifree representations of Clifford algebra // Publ. RIMS (Kyoto Univ. ). 1995. V. 31. P. 33-44. 